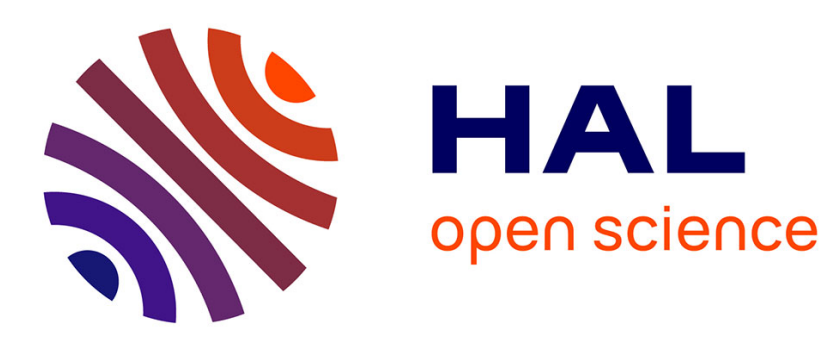

\title{
POINTS NON FACTORIELS DES VARIÉTÉS DE MODULES DE FAISCEAUX SEMI-STABLES SUR UNE SURFACE RATIONNELLE
}

\author{
Jean-Marc Drézet
}

\section{> To cite this version:}

Jean-Marc Drézet. POINTS NON FACTORIELS DES VARIÉTÉS DE MODULES DE FAISCEAUX SEMI-STABLES SUR UNE SURFACE RATIONNELLE. Journal für die reine und angewandte Mathematik, 1991, 290, pp.99-127. hal-01175951

\section{HAL Id: hal-01175951 \\ https://hal.science/hal-01175951}

Submitted on 13 Jul 2015

HAL is a multi-disciplinary open access archive for the deposit and dissemination of scientific research documents, whether they are published or not. The documents may come from teaching and research institutions in France or abroad, or from public or private research centers.
L'archive ouverte pluridisciplinaire HAL, est destinée au dépôt et à la diffusion de documents scientifiques de niveau recherche, publiés ou non, émanant des établissements d'enseignement et de recherche français ou étrangers, des laboratoires publics ou privés. 


\title{
POINTS NON FACTORIELS DES VARIÉTÉS DE MODULES DE FAISCEAUX SEMI-STABLES SUR UNE SURFACE RATIONNELLE
}

\author{
J.-M. DRÉZET
}

SOMMAIRE

1. Préliminaires 8

2. Caractérisation des points factoriels 14

\begin{tabular}{lll}
\hline 3. & Non-factorialité des points de type 2 & 18
\end{tabular}

4. Faisceaux universels 19

5. Étude d'un cas particulier 20

\begin{tabular}{ll}
\hline Références & 24
\end{tabular}

\section{0 - INTRODUCTION}

Soient $X$ une surface algébrique projective lisse et rationnelle sur $\mathbb{C}$ et $\mathcal{O}_{X}(1)$ un fibré en droites très ample sur $X$. On supposera que $K \cdot \mathcal{O}_{X}(1)<0, K$ désignant le fibré canonique sur $X$.

Pour tout faisceau algébrique cohérent $G$ sur $X, P_{G}$ désigne le polynôme de Hilbert de $G$ relativement à $\mathcal{O}_{X}(1)$. On dit qu'un faisceau algébrique cohérent $E$ sur $X$ est semi-stable (resp. stable) s'il est sans torsion et si pour tout sous-faisceau propre $F$ de $E$ on a

$$
\frac{P_{F}(m)}{\operatorname{rg}(F)} \leq \frac{P_{E}(m)}{\operatorname{rg}(E)} \quad(\text { resp. }<) \text { pour } m \gg 0 \text {. }
$$

Soit $H$ un polynôme à coefficients rationnels. On peut définir la variété de modules $M_{\mathcal{O}_{X}(1)}(H)$ des faisceaux semi-stables sur $X$ de polynôme de Hilbert $H$ relativement à $\mathcal{O}_{X}(1)$ (cf. [9], [13]). C'est une variété projective.

Le but principal de ce travail est d'étudier la factorialité des anneaux locaux des points de $M_{\mathcal{O}_{X}(1)}(H)$. On généralisera pour cela des résultats et des méthodes de [7]. On n'obtient pas de résultat complet comme dans le cas des courbes algébriques ([7]) ou de $\mathbb{P}_{2}([5])$, faute de connaître des propriétés qui sont pourtant a priori plus élémentaires de $M_{\mathcal{O}_{X}(1)}(H)$, par exemple quelles sont ses composantes irréductibles.

0.1 - Description de $M_{\mathcal{O}_{X}(1)}(H)$ (cf. [9], [13]). Si $E$ est un faisceau semi-stable sur $X$, il existe une filtration de Jordan-Hölder de $E$ :

$$
0=E_{0} \subset E_{1} \subset \cdots \subset E_{k}=E
$$


par des sous-faisceaux telle que $E_{i} / E_{i-1}$ soit stable et

$$
\frac{P_{E_{i}}}{\operatorname{rg}\left(E_{i}\right)}=\frac{P_{E}}{\operatorname{rg}(E)} \quad \text { pour } 1 \leq i \leq k
$$

Une telle filtration n'est pas nécessairement unique, mais la classe d'isomorphisme du gradué l'est, Plus précisément, si $0=E_{0}^{\prime} \subset E_{1}^{\prime} \subset \cdots \subset E_{k^{\prime}}^{\prime}=E$ est une autre filtration de Jordan-Hölder de $E$, on a $k^{\prime}=k$ et il existe une permutation $\sigma$ de $\{1, \ldots, k\}$ telle que $E_{i}^{\prime} / E_{i-1}^{\prime} \simeq E_{\sigma(i)} / E_{\sigma(i)-1}$ pour $1 \leq i \leq k$. On notera $\operatorname{Gr}(E)$ la classe d'isomorphisme de $\bigoplus_{1 \leq i \leq k} E_{i} / E_{i-1}$. On dit que deux faisceaux semi-stables $E, E^{\prime}$ sur $X$ sont $S$-équivalents si $\operatorname{Gr}(E)=\operatorname{Gr}\left(E^{\prime}\right)$. Les points fermés de $M_{\mathcal{O}_{X}(1)}(H)$ sont exactement les classes de $S$-équivalence de faisceaux semi-stables sur $X$ de polynôme de Hilbert $H$. L'ensemble des classes d'isomorphisme de faisceaux stables sur $X$ de polynôme de Hilbert $H$ est donc un sous-ensemble de $M_{\mathcal{O}_{X}(1)}(H)$. En fait, c'est un ouvert, noté $M_{\mathcal{O}_{X}(1)}^{s}(H)$.

Soit $E$ un faisceau semi-stable sur $X$ de polynôme de Hilbert $H$. On a alors

$$
H(m)=r\left(P\left(\mu+m \mathcal{O}_{X}(1)\right)-\Delta\right)
$$

avec

$$
r=\operatorname{rg}(E), \quad \mu=\mu(E)=\frac{c_{1}(E)}{r}, \quad \Delta=\Delta(E)=\frac{1}{r}\left(c_{2}(E)-\frac{r-1}{2 r} c_{1}(E)^{2}\right),
$$

$P$ étant le polynôme $P(\delta)=\frac{\delta(\delta-K)}{2}+1$, pour tout $\delta \in H^{2}(X, \mathbb{Z}) \otimes \mathbb{Q}$. Fixer $H$ revient donc à fixer $r, n=c_{1}(E) \cdot \mathcal{O}_{X}(1)$ et $\chi=H(0)$.

Soit $\Gamma$ l'ensemble des $\left(c_{1}, c_{2}\right) \in H^{2}(X, \mathbb{Z}) \times \mathbb{Z}$ tels qu'un faisceau de rang $r$ et de classes de Chern $c_{1}, c_{2}$ ait pour polynôme de Hilbert $H$. Alors $M_{\mathcal{O}_{X}(1)}(H)$ se décompose en union disjointe de sous-variétés fermées :

$$
M_{\mathcal{O}_{X}(1)}(H)=\coprod_{\left(c_{1}, c_{2}\right) \in \Gamma} M_{\mathcal{O}_{X}(1)}\left(r, c_{1}, c_{2}\right)
$$

les points fermés de $M_{\mathcal{O}_{X}(1)}\left(r, c_{1}, c_{2}\right)$ étant les classes de $S$-équivalence de faisceaux semi-stables sur $X$, de rang $r$ et de classes de Chern $c_{1}, c_{2}$. Il n'y a qu'un nombre fini de $\left(c_{1}, c_{2}\right) \in \Gamma$ tels que $M_{\mathcal{O}_{X}(1)}\left(r, c_{1}, c_{2}\right)$ soit non vide. Pour simplifier, on notera

$$
M\left(r, c_{1}, c_{2}\right)=M_{\mathcal{O}_{X}(1)}\left(r, c_{1}, c_{2}\right), \quad M^{s}\left(r, c_{1}, c_{2}\right)=M\left(r, c_{1}, c_{2}\right) \cap M_{\mathcal{O}_{X}(1)}^{s}(H),
$$

bien que ces variétés dépendent de $\mathcal{O}_{X}(1)$.

0.2 - Construction de $M\left(r, c_{1}, c_{2}\right)$ et caractérisation des points factoriels. Pour construire $M\left(r, c_{1}, c_{2}\right)$ on part d'un ouvert $R^{s s}$ d'un schéma de Grothendieck (cf. $\S 1.2$ ) sur lequel opère algébriquement un groupe du type $\operatorname{PGL}(p)=\mathrm{GL}(p) / \mathbb{C}^{*}$. ll existe un bon quotient $R^{s s} / \mathrm{PGL}(p)$ qui est justement $M\left(r, c_{1}, c_{2}\right)$. On note $\pi: R^{s s} \rightarrow M\left(r, c_{1}, c_{2}\right)$ le morphisme quotient.

L'hypothèse $K \cdot \mathcal{O}_{X}(1)<0$ entraine que $R^{s s}$ est lisse $\left([13]\right.$, §6). Il en découle que $M\left(r, c_{1}, c_{2}\right)$ est une variété normale. Le groupe $\operatorname{PGL}(p)$ agit librement sur $R^{s}=\pi^{-1}\left(M\left(r, c_{1}, c_{2}\right)\right)$, et la restriction de $\pi, R^{s} \rightarrow M^{s}\left(r, c_{1}, c_{2}\right)$ est un quotient géométrique. Il en découle que $M^{s}\left(r, c_{1}, c_{2}\right)$ est lisse. 
On se placera toujours dans le cas où $\operatorname{codim}_{R^{s s}}\left(R^{s s} \backslash R^{s}\right) \geq 2$ et où $M^{s}\left(r, c_{1}, c_{2}\right)$ est dense dans $M\left(r, c_{1}, c_{2}\right)$ (si tel n'est pas le cas on pourrait se limiter aux composantes de $M\left(r, c_{1}, c_{2}\right)$ où ces conditions sont vérifiées). Il en découle que $M\left(r, c_{1}, c_{2}\right) \backslash M^{s}\left(r, c_{1}, c_{2}\right)$ est précisément l'ensemble des points singuliers de $M\left(r, c_{1}, c_{2}\right)$ (la démonstration est la même que pour les variétés de modules de fibrés semi-stables sur les courbes algébriques ([17])).

Soit $U$ un ouvert non vide de $M\left(r, c_{1}, c_{2}\right)$. On appelle PGL $(p)$-fibré en droites sur $\pi^{-1}(U)$ un fibré en droites algébrique sur $\pi^{-1}(U)$ muni d'une action linéaire algébrique de $\operatorname{PGL}(p)$ compatible avec l'action de $\operatorname{PGL}(p)$ sur $\pi^{-1}(U)$. On démontrera le

Théorème $\mathbf{A}$ : Soient z un point fermé de $M\left(r, c_{1}, c_{2}\right)$, y un point de $R^{\text {ss }}$ au dessus de z tel que l'orbite $\mathrm{PGL}(p)$ y soit fermée. Alors l'anneau local de z est factoriel si et seulement si pour tout $\operatorname{PGL}(p)$-fibré en droites $L$ sur $R^{\text {ss }}$, le stabilisateur de y agit trivialement sur $L_{y}$.

C'est une généralisation de la méthode de démonstration de la factorialité locale des variétés de modules de fibrés semi-stables sur une courbe algébrique ([7]). Nous verrons aussi le lien entre les $\operatorname{PGL}(p)$-fibrés en droites sur $R^{s s}$ et le groupe de Picard du foncteur associé à $M\left(r, c_{1}, c_{2}\right)$ (cf. $§ 1.4)$.

0.2.1 - Soit $E$ un faisceau semi-stable dont la classe de $S$-équivalence est $z$. On peut écrire

$$
\operatorname{Gr}(E)=\left(E_{1} \otimes \mathbb{C}^{n_{1}}\right) \oplus \cdots \oplus\left(E_{k} \otimes \mathbb{C}^{n_{k}}\right),
$$

les $E_{i}$ étant des faisceaux stables deux à deux non isomorphes, Alors le stabilisateur de $y$ dans $\operatorname{PGL}(p)$ s'identifie à

$$
\operatorname{Aut}(\operatorname{Gr}(E)) / \mathbb{C}^{*} \simeq\left(\mathrm{GL}\left(n_{1}\right) \times \cdots \times \mathrm{GL}\left(n_{k}\right)\right) / \mathbb{C}^{*}
$$

et est donc parfaitement déterminé.

Pour $1 \leq i \leq k$, soient

$$
r_{i}=\operatorname{rg}\left(E_{i}\right), \quad c_{1 i}=c_{1}\left(E_{i}\right), \quad c_{2 i}=c_{2}\left(E_{i}\right) .
$$

On a un morphisme canonique

$$
\alpha: \prod_{1 \leq i \leq k} M\left(r_{i}, c_{1 i}, c_{2 i}\right) \longrightarrow M\left(r, c_{1}, c_{2}\right)
$$

associant à $\left(z_{1}, \ldots, z_{k}\right), z_{i}$ étant la classe de $S$-équivalence de $F_{i}$, celle de $\left(F_{i} \otimes \mathbb{C}^{n_{1}}\right) \oplus \cdots \oplus\left(F_{k} \otimes \mathbb{C}^{n_{k}}\right)$. Alors $M\left(r, c_{1}, c_{2}\right)$ est l'union disjointe finie des sous-ensembles du type

$$
\alpha\left(\prod_{1 \leq i \leq k} M\left(r_{i}, c_{1 i}, c_{2 i}\right)\right) .
$$

Soient $z, z^{\prime} \in M\left(r, c_{1}, c_{2}\right)$, avec $z \in \alpha(U), U$ étant une composante irréductible de $\prod_{1 \leq i \leq k} M\left(r_{i}, c_{1 i}, c_{2 i}\right)$. Alors on introduit une relation de préordre sur $M\left(r, c_{1}, c_{2}\right): z \geq z^{\prime}$ si et seulement si $z^{\prime} \in \overline{\alpha(U)}$. On démontrera le

Théorème B : Soient $z, z^{\prime}$ des points fermés de $M\left(r, c_{1}, c_{2}\right)$, avec $z \geq z^{\prime}$. Alors si $\mathcal{O}_{z^{\prime}}$ est factoriel, il en est de même de $\mathcal{O}_{z}$. 
En particulier, l'ensemble des points factoriels de $M\left(r, c_{1}, c_{2}\right)$ est ouvert.

0.3 - Exemples de points non factoriels. Soit $z \in M\left(r, c_{1}, c_{2}\right)$, correspondant à un faisceau semi-stable $E=E_{1} \oplus \cdots \oplus E_{k}$, avec $E_{i}$ stable pour $1 \leq i \leq k$. On dit que $z$ est de type 1 si on a

$$
\frac{c_{1}\left(E_{i}\right)}{\operatorname{rg}\left(E_{i}\right)}=\frac{c_{1}(E)}{\operatorname{rg}(E)} \quad \text { pour } 1 \leq i \leq k \quad\left(\text { dans } H^{2}(X, \mathbb{Q})\right)
$$

et de type 2 dans le cas contraire. Rappelons qu'on a $\frac{P_{E}}{\operatorname{rg}(E)}=\frac{P_{E_{i}}}{\operatorname{rg}\left(E_{i}\right)}$, ce qui équivaut à

$$
\frac{c_{1}\left(E_{i}\right) \cdot \mathcal{O}_{X}(1)}{\operatorname{rg}\left(E_{i}\right)}=\frac{c_{1}(E) \cdot \mathcal{O}_{X}(1)}{\operatorname{rg}(E)} \quad \text { et } \quad \frac{\chi\left(E_{i}\right)}{\operatorname{rg}\left(E_{i}\right)}=\frac{\chi(E)}{\operatorname{rg}(E)} \quad \text { pour } 1 \leq i \leq k .
$$

Théorème C : L'anneau local d'un point de type 2 de $M\left(r, c_{1}, c_{2}\right)$ n'est pas factoriel.

Pour démontrer ce résultat on utilise le théorème A et les exemples de PGL $(p)$-fibrés en droites sur $R^{s s}$ construits ci dessous.

0.4 - Groupe de Grothendieck de $X$ et PGL $(p)$-fibrés en droites sur $R^{s s}$. Sur $R^{s s} \times X$ existe un faisceau universel $\mathbb{E},(\mathrm{cf}$. $\$ 1.2)$ tel que le morphisme quotient $\pi: R^{s s} \rightarrow M\left(r, c_{1}, c_{2}\right)$ associe à $y$ la classe de $S$-équivalence de $\mathbb{E}_{y}$. Ce faisceau $\mathbb{E}$ est muni d'une action de $\operatorname{GL}(p)$ au dessus de l'action de PGL $(p)$ sur $R^{s s}$. L'action d'un élément $t$ de $\mathbb{C}^{*} \subset \operatorname{GL}(p)$ est simplement la multiplication par $t$.

Si $Y$ est une variété algébrique, on note $K(Y)$ le groupe de Grothendieck des faisceaux cohérents sur $Y$, isomorphe au groupe de Grothendieck des faisceaux localement libres sur $Y$ si $Y$ est lisse et quasi-projective. Si $E$ est un faisceau cohérent sur $Y$, on note $[E]$ la classe de $E$ dans $K(Y)$. Soient $p_{X}, p_{R}$ les projections de $R^{s s} \times X$ sur $X$ et $R^{s s}$ respectivement. Soient $E$ un faisceau cohérent de rang $r$ et de classes de Chern $c_{1}, c_{2}$, et $m$ le morphisme de groupes

$$
\begin{aligned}
K(X) \longrightarrow \mathbb{Z} \\
\alpha \longmapsto \chi([E] \otimes \alpha) .
\end{aligned}
$$

Ce morphisme ne dépend que de $r, c_{1}, c_{2}$. On pose $H\left(r, c_{1}, c_{2}\right)=\operatorname{ker}(m)$. Soit $\alpha \in K(X)$. Alors on définit successivement les éléments

$$
\begin{array}{rlll}
{[\mathbb{E}] \otimes p_{X}^{*}(\alpha)} & \text { de } & K\left(R^{s s} \times X\right), \\
p_{R !}\left([\mathbb{E}] \otimes p_{X}^{*}(\alpha)\right) & \text { de } & K\left(R^{s s}\right), \\
\mathbb{L}_{\alpha}=\operatorname{det}\left(p_{R !}\left([\mathbb{E}] \otimes p_{X}^{*}(\alpha)\right)\right) & \text { de } & \operatorname{Pic}\left(R^{s s}\right) .
\end{array}
$$

Le fibré $\mathbb{L}_{\alpha}$ est muni d'une action naturelle de $\operatorname{GL}(p)$ telle que l'action d'un élément $t$ de $\mathbb{C}^{*}$ soit la multiplication par $t^{m(\alpha)}$. Si $m(\alpha)=0, \mathbb{L}_{\alpha}$ est donc un PGL $(p)$-fibré en droites sur $R^{s s}$. Si $U$ est un ouvert non vide de $M\left(r, c_{1}, c_{2}\right)$, on note $\operatorname{Pic}^{G}\left(\pi^{-1}(U)\right)$ le groupe des classes d'isomorphisme de $\mathrm{PGL}(p)$-fibrés en droites sur $\pi^{-1}(U)$. On a donc un morphisme de groupes

$$
\mathbb{L}: H\left(r, c_{1}, c_{2}\right) \longrightarrow \operatorname{Pic}^{G}\left(R^{s s}\right) \text {. }
$$


On dit qu'un PGL $(p)$-fibré en droites $L$ sur $\pi^{-1}(U)$ descend à $U$ s'il existe un fibré en droites $L^{\prime}$ sur $U$ tel que les PGL $(p)$-fibrés en droites $\pi^{*}\left(L^{\prime}\right)$ et $L$ soient isomorphes. Pour qu'il en soit ainsi, il faut et il suffit que pour tout $y \in \pi^{-1}(U)$ tel que l'orbite $\operatorname{PGL}(p) y$ soit fermée, le stabilisateur de $y$ agisse trivialement sur $L_{y}$ (c'est une conséquence du "Lemme de descente", thm. 2.3 de [7]). C'est évidemment toujours le cas si $U=M^{s}\left(r, c_{1}, c_{2}\right)$. On en déduit un morphisme de groupes

$$
\gamma: H\left(r, c_{1}, c_{2}\right) \longrightarrow \operatorname{Pic}\left(M^{s}\left(r, c_{1}, c_{2}\right)\right) \text {. }
$$

La démonstration du théorème $\mathrm{C}$ est basée sur le fait que si $z$ est un point de type 2 , il existe des éléments de l'image de $\gamma$ qui ne se prolongent pas en fibrés en droites définis au voisinage de $z$.

Je conjecture que le morphisme $\gamma$ est surjectif. On sait que c'est vrai si $X=\mathbb{P}_{2}([5]$, thms. D et $\mathrm{E})$.

\section{5 - Points factorlels de $M\left(r, c_{1}, c_{2}\right)$}

Conjecture 1 : Les anneaux locaux des points de type 1 sont factoriels.

Deux méthodes sont a priori possibles pour démontrer cette conjecture. La première consiste à prouver la conjecture de 0.4 (concernant $\operatorname{Pic}\left(M^{s}\left(r, c_{1}, c_{2}\right)\right)$ ). Le résultat découlant alors du fait que tout PGL $(p)$-fibré en droites sur $R^{s s}$ venant de $H\left(r, c_{1}, c_{2}\right)$ descend à l'ouvert de $M\left(r, c_{1}, c_{2}\right)$ constitué des points de type 1.

L'autre méthode consiste à utiliser les théorèmes A et B. Il suffit en effet de montrer que si $z$ est un point de type 1 , il existe un point $z^{\prime}$ tel que $z \geq z^{\prime}$ et que $z^{\prime}$ soit la classe de $S$ équivalence d'un faisceau du type $F^{\prime}=E^{\prime} \otimes \mathbb{C}^{n}$, avec $E^{\prime}$ stable. L'anneau $\mathcal{O}_{z^{\prime}}$ est factoriel d'après le théorème $\mathrm{A}$, car $\operatorname{Aut}\left(F^{\prime}\right) / \mathbb{C}^{*}$ est isomorphe à $\operatorname{PGL}(n)$, et n'a pas de caractère non trivial. Donc $\mathcal{O}_{z}$ est aussi factoriel d'après le théorème B.

Examinons ce que signifie l'existence d'un tel $z^{\prime}$. Supposons que $z$ soit la classe de $S$-équivalence d'un faisceau semi-stable de la forme

$$
E=E_{1} \oplus \cdots \oplus E_{k}, \quad k \geq 2,
$$

les $E_{i}$ étant des faisceaux stables, avec

$$
\frac{c_{1}\left(E_{i}\right)}{\operatorname{rg}\left(E_{i}\right)}=\frac{c_{1}}{r}=\mu, \quad \text { pour } 1 \leq i \leq k .
$$

Il existe alors des entiers $n_{i} \geq 1, \chi_{0}, r_{0}$, et un élément $c_{10}$ de $H^{2}(X, \mathbb{Z})$ tels que pour $1 \leq i \leq k$ on ait

Alors

$$
c_{1}\left(E_{i}\right)=n_{i} c_{10}, \quad \operatorname{rg}\left(E_{i}\right)=n_{i} r_{0}, \quad \chi\left(E_{i}\right)=n_{i} \chi_{0} .
$$

$$
c_{20}=r_{0} P(\mu)-\chi_{0}+\frac{r_{0}\left(r_{0}-1\right)}{2} \mu^{2}
$$

est un entier (c'est la seconde classe de Chern d'un faisceau $F$ tel que $\operatorname{rg}(F)=r_{0}, c_{1}(F)=c_{10}$, $\left.\chi(F)=\chi_{0}\right)$. Il est aisé de voir que l'existence de $z^{\prime}$ sera démontrée si on prouve les deux assertions suivantes :

(i) $M^{s}\left(r_{0}, c_{10}, c_{20}\right)$ est non vide.

(ii) Pour $1 \leq i \leq k, M\left(\operatorname{rg}\left(E_{i}\right), c_{1}\left(E_{i}\right), c_{2}(E i)\right)$ est irréductible. 
L'assertion (ii) connait un début de démonstration (Ballico [1]).

L'assertion (i) est un cas particulier de la conjecture suivante, inspirée de ce qui se passe sur $\mathbb{P}_{2}([6],[3]):$

Conjecture 2 : Il existe une unique fonction $\delta: H^{2}(X, \mathbb{Z}) \otimes \mathbb{Q} \longrightarrow \mathbb{Q}$ (dépendant de $\mathcal{O}_{X}(1)$ ) telle que pour tous entiers $r, c_{2}$, avec $r \geq 1$, et tout $c_{1} \in H^{1}(X, \mathbb{Z})$, si

$$
\mu=\frac{c_{1}}{r}, \quad \Delta=\frac{1}{r}\left(c_{2}-\frac{r-1}{2 r} c_{1}^{2}\right),
$$

on ait $\operatorname{dim}\left(M^{s}\left(r, c_{1}, c_{2}\right)>0\right.$ si et seulement si $\Delta \geq \delta\left(r, c_{1}, c_{2}\right)$.

En effet, dans le cas qui nous préoccupe, on a

$$
\frac{c_{10}}{r_{0}}=\frac{c_{1}}{r} \quad \text { et } \frac{1}{r_{0}}\left(c_{20}-\frac{r_{0}-1}{r_{0}} c_{10}^{2}\right)=\frac{1}{r}\left(c_{2}-\frac{r-1}{2 r} c_{1}^{2}\right),
$$

donc si la conjecture 2 est vraie, on a

$$
\operatorname{dim}\left(M^{s}\left(r, c_{1}, c_{2}\right)\right)>0 \Longrightarrow \operatorname{dim}\left(M^{s}\left(r_{0}, c_{10}, c_{20}\right)\right)>0 .
$$

Pour résumer, on peut dire que la démonstration de la conjecture 1 par la seconde méthode passe par la découverte des conditions d'existence des faisceaux semi-stables de rang et classes de Chern donnés, et par la preuve de l'irréductibilité de ces variétés.

0.6 - Faisceaux universels. Dans ce qui précède, certains points de $M\left(r, c_{1}, c_{2}\right)$ jouent un rôle particulier dans la démonstration de la factorialité des anneaux locaux des points de type 1. Ce sont les points associés aux faisceaux du type $E \otimes \mathbb{C}^{n}, E$ étant stable, et $n \geq 2$. De tels points seront dits spéciaux. Ce sont des points de type 1.

On suppose dans ce qui suit que $M\left(r, c_{1}, c_{2}\right)$ est irréductible.

Soit $U$ un ouvert non vide de $M^{s}\left(r, c_{1}, c_{2}\right)$. Un faisceau de Poincaré sur $U$ est un faisceau cohérent $E$ sur $U \times X$, plat sur $U$, et tel que pour tout point fermé $z$ de $U, E_{z}=E_{\mid\{z\} \times X}$ soit stable et que sa classe d'isomorphisme soit précisément $z$.

Soit $\chi=r(P(\mu)-\Delta)$ (la caractéristique d'Euler-Poincaré des faisceaux de rang $r$ et de classes de Chern $\left.c_{1}, c_{2}\right)$. On note $a$ le plus grand entier tel que $c_{1}$ soit divisible par $a$ dans $H^{2}(X, \mathbb{Z})$ si $c_{1} \neq 0$, et si $c_{1}=0$ on prend $a=0$. Soit $d=\operatorname{PGCD}(r, a, \chi)$.

Théorème D : Soit $U$ un ouvert non vide de $M^{s}\left(r, c_{1}, c_{2}\right)$.

(1) Si $M\left(r, c_{1}, c_{2}\right)$ contient un point spécial, alors il n'existe pas de faisceau de Poincaré sur $U$.

(2) Si $d=1$, alors il existe un faisceau de Poincaré sur $U$.

On voit aisément que si la conjecture 2 est vraie, et si $d \neq 1$, alors $M\left(r, c_{1}, c_{2}\right)$ contient un point spécial. Si cette conjecture est prouvée on sait donc quels sont les cas ou un faisceau de Poincaré existe. 
On remarquera qu'il est possible que $d=1$ et $M\left(r, c_{1}, c_{2}\right) \neq M^{s}\left(r, c_{1}, c_{2}\right)$ (on peut trouver des exemples sur $\mathbb{P}_{1} \times \mathbb{P}_{1}$. Même dans ce cas il existe un faisceau de Poincaré sur $M^{s}\left(r, c_{1}, c_{2}\right)$. Le théorème $\mathrm{D}$ permet de retrouver le résultat de Le Potier ([12], th. 1 et 2) concernant les familles universelles de fibrés stables de rang 2 sur $\mathbb{P}_{2}$.

0.7 - Le cas $X=\mathbb{P}_{1} \times \mathbb{P}_{1}, r=2, c_{1}=0$. Dans ce cas, on verra que si $M\left(2,0, c_{2}\right)$ est non vide, on a $c_{2} \geq 2$, et la condition $\operatorname{codim}_{M\left(r, c_{1}, c_{2}\right)}\left(M\left(r, c_{1}, c_{2}\right) \backslash M^{s}\left(r, c_{1}, c_{2}\right)\right) \geq 2$ équivaut à $c_{2}>2$. Les variétés $M\left(2,0, c_{2}\right)$ sont irréductibles.

Soient $p_{1}, p_{2}$ les projections $\mathbb{P}_{1} \times \mathbb{P}_{1} \rightarrow \mathbb{P}_{1}$. Alors Pic $\left(\mathbb{P}_{1} \times \mathbb{P}_{1}\right)$ est constitué des fibrés de la forme

$$
p_{1}^{*}\left(\mathcal{O}_{\mathbb{P}_{1}}(\alpha)\right) \otimes p_{2}^{*}\left(\mathcal{O}_{\mathbb{P}_{1}}(\beta)\right)=\mathcal{O}(\alpha, \beta) .
$$

Le fibré $\mathcal{O}(\alpha, \beta)$ est très ample si et seulement si $\alpha>0$ et $\beta>O$. Posons $\mathcal{O}_{X}(1)=\mathcal{O}(\alpha, \beta)$. On peut supposer que $\alpha$ et $\beta$ sont premiers entre eux.

Pour tout nombre réel $x$, on note $[x]$ la partie entière de $x$. On suppose que $c_{2}>2$. Alors $M\left(r, c_{1}, c_{2}\right) \neq M^{s}\left(r, c_{1}, c_{2}\right)$ si et seulement si $c_{2}$ est pair. Dans ce cas $M\left(r, c_{1}, c_{2}\right) \backslash M^{s}\left(r, c_{1}, c_{2}\right)$ comporte $m+1$ composantes connexes irréductibles $Z_{0}, \ldots, Z_{m}$, avec

$$
m=\left[\frac{-|\beta-\alpha|+\sqrt{(\alpha-\beta)^{2}+2 \alpha \beta c_{2}}}{2 \alpha \beta}\right] .
$$

La composante $Z_{k}$ est constituée des classes de $S$-équivalence des faisceaux de la forme

$$
I_{Y}(k \alpha,-k \beta) \oplus I_{Y^{\prime}}(-k \alpha, k \beta),
$$

ou $Y, Y^{\prime}$ sont des sous-schémas de dimension 0 de $\mathbb{P}_{1} \times \mathbb{P}_{1}$, de longueurs respectives $l(Y), \operatorname{ll}\left(Y^{\prime}\right)$, $I_{Y}, I_{Y^{\prime}}$ désignant leurs faisceaux d'idéaux, avec

$$
l(Y)=\frac{c_{2}}{2}-k^{2} \alpha \beta+k(\alpha-\beta), \quad l\left(Y^{\prime}\right)=\frac{c_{2}}{2}-k^{2} \alpha \beta+k(\beta-\alpha) .
$$

Les points de type 1 sont ceux de $M^{s}\left(2,0, c_{2}\right) \cup Z_{0}$. Les points de type 2 sont ceux de $Z_{1} \cup \cdots Z_{k}$. On a

$$
\operatorname{dim}\left(Z_{k}\right)=2\left(c_{2}-2 k^{2} \alpha \beta\right) .
$$

On verra que la conjecture 1 de 0.5 est vraie pour $M\left(2,0, c_{2}\right)$. Autrement dit, les points factoriels de $M\left(2,0, c_{2}\right)$ sont exactement les points de type 1 .

Remarque : $\operatorname{Si} \alpha=\beta=1$, et si $z$ est le point de $M(2,0,2)$ correspondant à $\mathcal{O}(-1,1) \oplus \mathcal{O}(1,-1)$, alors $\mathcal{O}_{z}$ n'est pas factoriel. Cet exemple de point non factoriel, dû à J. Le Potier, a motivé le présent article.

0.8 - Complétés des anneaux locaux des point de $M\left(r, c_{1}, c_{2}\right)$. On ne suppose plus ici que $X=\mathbb{P}_{1} \times \mathbb{P}_{1}$. Soit $z$ un point singulier de $M\left(r, c_{1}, c_{2}\right)$. Ce point est la classe de $S$-équivalence d'un faisceau du type

$$
E=E_{1} \oplus \cdots \oplus E_{k},
$$

avec $k \geq 2$, les $E_{i}$ étant stables. Sur $\operatorname{Ext}^{1}(E, E)$ agit le groupe réductif $\operatorname{Aut}(E)$. Il existe un bon quotient $\operatorname{Ext}^{1}(E, E) / \operatorname{Aut}(E)=N$. Soit $x$ l'image de 0 dans $N$. Comme dans [4], $\S 9$, on 
montre que le complété de l'anneau local $\mathcal{O}_{z}$ est isomorphe à celui de $\mathcal{O}_{N, x}$. Ceci montre qu'il n'est pas possible en général de décider de la factorialité de $\mathcal{O}_{z}$ en examinant seulement son complété.

Par exemple, supposons que $X=\mathbb{P}_{1} \times \mathbb{P}_{1}, \mathcal{O}_{X}(1)=\mathcal{O}(1,1)$. Considérons l'action de $\mathbb{C}^{*} \times \mathbb{C}^{*}$ sur $\mathbb{C}^{10}$ :

$$
\left((\lambda, \mu),\left(\alpha_{i}\right)_{1 \leq i \leq 10}\right) \mapsto\left(\frac{\lambda}{\mu} \alpha_{1}, \ldots, \frac{\lambda}{\mu} \alpha_{5}, \frac{\mu}{\lambda} \alpha_{6}, \ldots, \frac{\mu}{\lambda} \alpha_{10}\right) .
$$

et soient $N=\mathbb{C}^{10} /\left(\mathbb{C}^{*} \times \mathbb{C}^{*}\right), x$ l'image de 0 dans $N$. Soient $z_{0}$ un point général de la composante $Z_{0}$ de $M(2,0,6), z_{1}$ un point de la composante $Z_{1}$ de $M(2,0,4)$. Alors on a

$$
\widehat{\mathcal{O}}_{z_{0}} \simeq \widehat{\mathcal{O}}_{N \times \mathbb{C}^{12},(x, 0)}, \quad \widehat{\mathcal{O}}_{z_{1}} \simeq \widehat{\mathcal{O}}_{N \times \mathbb{C}^{4},(x, 0)}
$$

Cependant, $\mathcal{O}_{z_{0}}$ est factoriel, alors que $\mathcal{O}_{z_{1}}$ ne l'est pas.

Notations. Si $X_{1}, \ldots, X_{p}$ sont des ensembles, on notera $p_{X_{i}}$ la projection $X_{1} \times \cdots \times X_{p} \rightarrow X_{i}$.

Si $f: S^{\prime} \rightarrow S$ est un morphisme de variétés algébriques, et $F$ un faisceau cohérent sur $S \times X$, on posera

$$
f^{\#}(F)=(f \times I d)^{*}(F) .
$$

Si $E$ est un faisceau cohérent sur une variété algébrique projective $Y$, on notera

$$
H^{i}(E)=H^{i}(Y, E), \quad h^{i}(E)=\operatorname{dim}_{\mathbb{C}}\left(H^{i}(Y, E)\right) \text { pour tout entier } i .
$$

Soit $m$ un entier $\geq 1$. On notera $m E$ le faisceau $E \oplus \cdots \oplus E$, somme directe de $m$ copies de E.

\section{PRÉliminaires}

1.1 - Variétés de modules de faisceaux semi-stables. Soit $T$ une variété algébrique. Une famille de faisceaux de $M\left(r, c_{1}, c_{2}\right)$ paramétrée par $T$ est un faisceau cohérent $E$ sur $T \times X$, plat sur $T$, tel que pour tout point fermé $t$ de $T, E_{t}=E_{\mid\{t\} \times X}$ soit semi-stable, de rang $r$ et de classes de Chern $c_{1}, c_{2}$. Deux telles familles $E, E^{\prime}$ sont dites équivalentes s'il existe un fibré en droites $L$ sur $T$ et un isomorphisme $E^{\prime} \simeq E \otimes p_{T}^{*}(L)$.

On note $F\left(r, c_{1}, c_{2}\right)$ le foncteur

\section{Variétés algébriques $\longrightarrow$ Ensembles}

associant à $T$ l'ensemble des classes d'équivalence de familles de faisceaux de $M\left(r, c_{1}, c_{2}\right)$ paramétrées par T. Si $f: T^{\prime} \rightarrow T$ est un morphisme de variétés algébriques, et si $E$ est une famille de faisceaux de $M\left(r, c_{1}, c_{2}\right)$ paramétrée par $T$, l'image par $F\left(r, c_{1}, c_{2}\right)(f)$ de la classe de $E$ est celle de $f^{\#}(E)$. Il existe un morphisme canonique de foncteurs

$$
\Psi: F\left(r, c_{1}, c_{2}\right) \longrightarrow \operatorname{Hom}\left(\bullet, M\left(r, c_{1}, c_{2}\right)\right) .
$$

À toute famille $\mathrm{E}$ de faisceaux de $M\left(r, c_{1}, c_{2}\right)$ paramétrée par $T$ on associe donc un morphisme de variétés

$$
f_{E}: T \longrightarrow M\left(r, c_{1}, c_{2}\right)
$$


associant au point fermé $t$ de $T$ la classe de $S$-équivalence de $E_{t}$.

La variété $M\left(r, c_{1}, c_{2}\right)$ est caractérisée par la propriété suivante : si $M$ est une variété algébrique et

$$
\Psi^{\prime}: F\left(r, c_{1}, c_{2}\right) \longrightarrow \operatorname{Hom}(\bullet, M)
$$

un morphisme de foncteurs, il existe un unique morphisme

$$
f: M\left(r, c_{1}, c_{2}\right) \longrightarrow M
$$

tel que $\Psi^{\prime}=\operatorname{Hom}(\bullet, f) \circ \Psi$.

1.2 - Construction de $M\left(r, c_{1}, c_{2}\right)$. Il existe un entier $m_{0}$ tel que pour tout entier $m \geq m_{0}$, et tout faisceau semi-stable $E$ sur $X$ de rang $r$ et de classes de Chern $c_{1}, c_{2}, E(m)=E \otimes \mathcal{O}_{X}(m)$ soit engendré par ses sections, et $h^{i}(E(m))=0$ pour $i>0$. Fixons un $m \geq m_{0}$. On pose $p=h^{0}(E(m))=\chi(E(m))$, qui ne dépend pas du choix de $E$. On rappelle que $H$ désigne le polynôme de Hilbert de $E$. Soit

$$
R=\operatorname{Quot}_{H}\left(\mathcal{O}_{X}(-m) \otimes \mathbb{C}^{p}\right)
$$

C'est la variété projective représentant le foncteur

$$
\Psi_{0} \text { : Variétés algébriques } \longrightarrow \text { Ensembles }
$$

associant à $T$ l'ensemble des classes d'isomorphisme de morphismes surjectifs de faisceaux sur $T \times X$

$$
p_{X}^{*}\left(\mathcal{O}_{X}(-m)\right) \otimes \mathbb{C}^{p} \longrightarrow E,
$$

où $E$ est plat sur $T$, et pour tout point fermé $t$ de $T, E_{t}$ a pour polynôme de Hilbert $H$ relativement à $\mathcal{O}_{X}(1)$ (deux tels morphismes

$$
f: p_{X}^{*}\left(\mathcal{O}_{X}(-m)\right) \otimes \mathbb{C}^{p} \longrightarrow E \quad \text { et } \quad f^{\prime}: p_{X}^{*}\left(\mathcal{O}_{X}(-m)\right) \otimes \mathbb{C}^{p} \longrightarrow E^{\prime}
$$

sont isomorphes s'il existe un isomorphisme $\phi: E \rightarrow E^{\prime}$ tel qu'on ait $\left.f^{\prime}=\phi \circ f\right)$. Il existe un morphisme universel surjectif

$$
\Theta: p_{X}^{*}\left(\mathcal{O}_{X}(-m)\right) \otimes \mathbb{C}^{p} \longrightarrow \mathbb{E}
$$

sur $R \times X$ (cf. [10]). On note $R^{s s}$ (resp. $R^{s}$ ) l'ouvert des points $y$ de $R$ tels que

$$
\Theta_{y}: \mathcal{O}_{X}(-m) \otimes \mathbb{C}^{p} \longrightarrow \mathbb{E}_{y}
$$

induise un isomorphisme $\mathbb{C}^{p} \simeq H^{0}\left(\mathbb{E}_{y}(m)\right.$ ), et que $\mathbb{E}_{y}$ soit semi-stable (resp. stable), de rang $r$ et de classes de Chern $c_{1}, c_{2}$.

La condition $K . \mathcal{O}_{X}(1)<0$ entraine que $R^{s s}$ est lisse (cf. [13], §6). De la restriction de $\mathbb{E}$ à $R^{s s} \times X$, qui est une famille de faisceaux de $M\left(r, c_{1}, c_{2}\right)$ paramétrée par $R^{s s}$, on déduit un morphisme

$$
\pi=f_{\mathbb{E}}: R^{s s} \longrightarrow M\left(r, c_{1}, c_{2}\right) .
$$

Le groupe $\operatorname{PGL}(p)$ agit de façon naturelle sur $R^{s s}$. Explicitons cette action. Les points fermés de $R^{s s}$ sont exactement les classes d'équivalence de morphismes surjectifs

$$
\phi: \mathcal{O}_{X}(-m) \otimes \mathbb{C}^{p} \longrightarrow E,
$$

$E$ étant un faisceau semi-stable de rang $r$ et de classes de Chern $c_{1}, c_{2}$ sur $X, \phi$ induisant un isomorphisme $\mathbb{C}^{p} \simeq H^{0}(E(m))$. Deux tels morphismes $\phi: \mathcal{O}_{X}(-m) \otimes \mathbb{C}^{p} \rightarrow E$ et $\phi^{\prime}: \mathcal{O}_{X}(-m) \otimes \mathbb{C}^{p} \longrightarrow E^{\prime}$ sont équivalents s'il existe un isomorphisme $\sigma: E \rightarrow E^{\prime}$ tel que 
$\phi^{\prime}=\sigma \circ \phi$. Soit $y$ le point de $R^{s s}$ correspondant à $\phi$, et $g \in \operatorname{GL}(p)$. Alors $g y$ est le point de $R^{s s}$ correspondant à

$$
\mathcal{O}_{X}(-m) \otimes \mathbb{C}^{p} \stackrel{g^{-1}}{\longrightarrow} \mathcal{O}_{X}(-m) \otimes \mathbb{C}^{p} \stackrel{\phi}{\longrightarrow} E
$$

Il est clair que si $g$ est une homothétie, on a $g y=y$. On obtient donc une action de $\operatorname{PGL}(p)$ sur $R^{s s}$.

On montre que si $m$ est assez grand, $\pi$ est un bon quotient de $R^{s s}$ par $\operatorname{PGL}(p)$, tandis que la restriction de $\pi: R^{s} \rightarrow M^{s}\left(r, c_{1}, c_{2}\right)$ est un quotient géométrique (cf. [13]).

Le stabilisateur d'un point fermé $y$ de $R^{s s}$ dans GL $(p)$ s'identifie naturellement au groupe des automorphismes de $\mathbb{E}_{y}$.

1.3 - Descente de fibrés vectoriels. On rappelle ici le "lemme de descente" ([7], thm. 2.3).

Soit $Y$ une variété algébrique intègre sur laquelle opère algébriquement un groupe algébrique réductif $G$. On suppose qu'il existe un bon quotient $\pi: Y \rightarrow M$ (cf. [16],[18]). Si $E$ est un $G$-fibré vectoriel sur $Y$, on dit que $E$ descend à $M$ s'il existe un fibré vectoriel $E^{\prime}$ sur $Y$ tel que les $G$-fibrés $E$ et $\pi^{*}\left(E^{\prime}\right)$ soient isomorphes.

Théorème 1.1 (Lemme de descente) : Soit $E$ un G-fibré vectoriel sur $Y$. Alors $E$ descend à $M$ si et seulement si pour tout point fermé y de $Y$ tel que Gy soit fermée, le stabilisateur de $y$ dans $G$ agit trivialement sur $E_{y}$.

1.4 - Groupe de Picard de $F\left(r, c_{1}, c_{2}\right)$ et PGL $(p)$-fibrés en droites sur $R^{s s}$. Les définitions données ici sont analogues à celles de [7], §3. On pourrait en fait les étendre à un cadre plus général. On ne donnera pas les démonstrations des énoncés qui vont suivre car elles sont identiques à celles des énoncés analogues de [7], §3.

Définition 1. Un fibré en droites $L$ sur $F\left(r, c_{1}, c_{2}\right)$ est défini par la donnée de

(i) Pour toute famille $E$ de faisceaux de $M\left(r, c_{1}, c_{2}\right)$ paramétrée par une variété lisse $S$, d'un fibré en droites $L_{E}$ sur $S$, ne dépendant que de la classe d'équivalence de $E$.

(ii) Pour tout morphisme $f: S^{\prime} \rightarrow S$ de variétés lisses, d'un isomorphisme

$$
\alpha_{E}^{L}(f): L_{f \#(E)} \longrightarrow f^{*}\left(L_{E}\right)
$$

ne dépendant que de la classe d'équivalence de $E$, tel que si $g: S^{\prime \prime} \rightarrow S^{\prime}$ est un autre morphisme de variétés lisses on ait

$$
\alpha_{E}^{L}(f \circ g)=g^{*}\left(\alpha_{E}^{L}(f)\right) \circ \alpha_{f \#(E)}^{L}(g) .
$$

Définition 2. Soient $L, L^{\prime}$ des fibrés en droites sur $F\left(r, c_{1}, c_{2}\right)$. Un isomorphisme $L \simeq L^{\prime}$ est la donnée, pour toute famille $E$ de faisceaux de $M\left(r, c_{1}, c_{2}\right)$ paramétrée par une variété lisse $S$, d'un isomorphisme

$$
\sigma_{E}: L_{E} \longrightarrow L_{E}^{\prime}
$$


ne dépendant que de la classe d'équivalence de $E$, tel que si $f: S^{\prime} \rightarrow S$ est un morphisme de variétés lisses, on ait

$$
\sigma_{f \#(E)}=\alpha_{E}^{L^{\prime}}(f)^{-1} \circ f^{*}\left(\sigma_{E}\right) \circ \alpha_{E}^{L}(f)
$$

Définition 3. Les classes d'isomorphisme de fibrés en droites sur $F\left(r, c_{1}, c_{2}\right)$ constituent de façon évidente un groupe commutatif, appelé groupe de Picard de $F\left(r, c_{1}, c_{2}\right)$ et noté $\operatorname{Pic}\left(F\left(r, c_{1}, c_{2}\right)\right)$.

Soit $L_{0}$ un fibré en droites sur $M\left(r, c_{1}, c_{2}\right)$. On en déduit un fibré en droites $L$ sur $F\left(r, c_{1}, c_{2}\right)$ défini par

$$
L_{E}=f_{E}^{\#}\left(L_{0}\right)
$$

pour toute famille $E$ de faisceaux de $M\left(r, c_{1}, c_{2}\right)$ paramétrée par une variété lisse, les $\alpha_{E}^{L}(f)$ étant définis de manière évidente. On obtient ainsi un morphisme de groupes

$$
i: \operatorname{Pic}\left(M\left(r, c_{1}, c_{2}\right)\right) \longrightarrow \operatorname{Pic}\left(F\left(r, c_{1}, c_{2}\right)\right) \text {. }
$$

Définition 4. On dit qu'un élément de $\operatorname{Pic}\left(F\left(r, c_{1}, c_{2}\right)\right)$ provient de $M\left(r, c_{1}, c_{2}\right)$ s'il est dans l'image de $i$.

Soit $L$ un fibré en droites sur $F\left(r, c_{1}, c_{2}\right)$. Alors le fibré $L_{\mathbb{E}}$ sur $R^{s s}$ est muni d'une action de $\operatorname{PGL}(p)$. Elle est définie par un isomorphisme

$$
\eta^{*}\left(p_{R^{s s}}^{*}\left(L_{\mathbb{E}}\right)\right) \simeq p_{R^{s s}}^{*}\left(L_{\mathbb{E}}\right)
$$

$\eta$ étant le morphisme

$$
\begin{gathered}
R^{s s} \times \operatorname{PGL}(p) \longrightarrow R^{s s} \times \operatorname{PGL}(p) \\
(y, g) \longmapsto(g y, g) .
\end{gathered}
$$

Cet isomorphisme provient du fait que les familles $p_{R^{s s}}^{*}\left(L_{\mathbb{E}}\right)$ et $\eta^{*}\left(p_{R^{s s}}^{*}\left(L_{\mathbb{E}}\right)\right)$ de faisceaux de $M\left(r, c_{1}, c_{2}\right)$ paramétrées par $R^{s s} \times \operatorname{PGL}(p)$ sont équivalentes (lemme 3.1 de [7], on utilise ici les hypothèses que $M^{s}\left(r, c_{1}, c_{2}\right)$ est dense dans $M\left(r, c_{1}, c_{2}\right)$ et que son complémentaire est de codimension au moins 2). On obtient donc un morphisme de groupes

$$
\operatorname{Pic}\left(F\left(r, c_{1}, c_{2}\right)\right) \longrightarrow \operatorname{Pic}^{G}\left(R^{s s}\right) \text {. }
$$

Lemme 1.2 : Le morphisme de groupes

$$
\begin{aligned}
\operatorname{Pic}\left(F\left(r, c_{1}, c_{2}\right)\right) \longrightarrow \operatorname{Pic}^{G}\left(R^{s}\right) \\
L \longmapsto L_{\mathbb{E} \mid R^{s}}
\end{aligned}
$$

est injectif.

(Prop. 3.3 et cor. 3.4 de [7].)

Remarquons que le morphisme d'oubli $\operatorname{Pic}^{G}\left(R^{s}\right) \rightarrow \operatorname{Pic}\left(R^{s}\right)$ est aussi injectif (cela découle du fait que $\operatorname{PGL}(p)$ n'a pas de caractère non trivial). 
Lemme 1.3 : Soit $L$ un fibré en droites sur $F\left(r, c_{1}, c_{2}\right)$. Alors $L$ provient de $M\left(r, c_{1}, c_{2}\right)$ si et seulement si $L_{\mathbb{E}}$ descend à $M\left(r, c_{1}, c_{2}\right)$. Dans ce cas, si $L_{0}$ est l'unique élément de $\operatorname{Pic}\left(M\left(r, c_{1}, c_{2}\right)\right)$ tel que $\pi^{*}\left(L_{0}\right) \simeq L$, on a $L=i\left(L_{0}\right)$.

(Cor. 3.5 de [7].)

1.5 - Groupe de Grothendieck de X. D'après Fulton ([8], 15.3.6), on a une filtration de $K(X)$ :

$$
O=F^{3} X \subset F^{2} X \subset F^{1} X \subset F^{0} X=K(X),
$$

telle qu'on ait des isomorphismes

$$
\begin{aligned}
\text { rang }: & F^{0} X / F^{1} X \rightarrow A^{0}(X) \\
\operatorname{det}: & F^{1} X / F^{2} X \longrightarrow A^{1}(X)=\operatorname{Pic}(X), \\
c_{2}: & F^{2} X / F^{1} X \longrightarrow A^{2}(X) .
\end{aligned}
$$

Puisque $A^{0}(X), A^{1}(X)$ et $A^{2}(X)$ sont sans torsion, on a donc un isomorphisme $K(X) \simeq A^{*}(X)$. Cela signifie qu'un élément de $K(X)$ est entièrement déterminé par ses rang et classes de Chern, et que tout triplet $\left(r, c_{1}, c_{2}\right)$ dans $\mathbb{Z} \times A^{1}(X) \times \mathbb{Z}$ peut s'écrire $\left(r, c_{1}, c_{2}\right)=\left(\operatorname{rg}(\alpha), c_{1}(\alpha), c_{2}(\alpha)\right)$ pour un $\alpha \in K(X)$.

1.6 - Exemples fondamentaux d'éléments de $\operatorname{Pic}\left(F\left(r, c_{1}, c_{2}\right)\right)$. Rappelons que $H\left(r, c_{1}, c_{2}\right)$ désigne le sous-groupe de $K(X)$ constitué des $\alpha$ tels que $\chi(\alpha \otimes[E])=0$ pour un, et donc tous les faisceaux cohérents $E$ de rang $r$ et de classes de Chern $c_{1}, c_{2}$ sur $X$. Cela signifie, si $R=\operatorname{rg}(\alpha), \beta_{i}=c_{i}(\alpha)$ pour $i=1,2$, que

$$
R c_{1}^{2}-R K c_{1}+r \beta_{1}^{2}-r K \beta_{1}+2 c_{1} \beta_{1}+2 r R-2 r \beta_{2}-2 R c_{2}=0 .
$$

Soit $\alpha \in H\left(r, c_{1}, c_{2}\right)$. On va en déduire un élément $\gamma(\alpha)$ de $\operatorname{Pic}\left(F\left(r, c_{1}, c_{2}\right)\right)$. Si $S$ est une variété algébrique lisse, et $E$ une famille de faisceaux de $M\left(r, c_{1}, c_{2}\right)$ paramétrée par $S$, on aura

$$
\gamma(\alpha)_{E}=\operatorname{det}\left(p_{S !}\left([E] \otimes p_{X}^{*}(\alpha)\right)\right) .
$$

Ce fibré ne dépend que de la classe d'équivalence de $E$ : si $L$ est un fibré en droites sur $S$, on a

$$
\gamma(\alpha)_{E \otimes p_{X}^{*}(L)}=\gamma(\alpha)_{E} \otimes L^{\chi\left(\left[E_{s}\right] \otimes \alpha\right)},
$$

$s$ étant un point quelconque de $S$. Puisque $\chi\left(\left[E_{s}\right] \otimes \alpha\right)=0$, on a $\gamma(\alpha)_{E \otimes p_{X}^{*}(L)}=\gamma(\alpha)_{E}$.

On ne peut pas déduire de $\alpha$ un fibré en droites sur $F\left(r, c_{1}, c_{2}\right)$. Pour en obtenir un, on considère une représentation de $\alpha$ sous la forme d'une somme finie

$$
\alpha=\sum n_{i}\left[F_{i}\right]
$$

les $n_{i}$ étant des entiers et les $F_{i}$ des fibrés vectoriels sur $X$. On pose

$$
L_{E}=\bigotimes_{i} \operatorname{det}\left(p_{S !}\left(E \otimes p_{X}^{*}\left(F_{i}\right)\right)^{n_{i}}\right.
$$

Les $\alpha_{E}^{L}(f)$ sont définis de manière évidente, et il est aisé de voir que si on choisit une représentation différente de $\alpha$, on obtient un fibré en droites sur $F\left(r, c_{1}, c_{2}\right)$ isomorphe à $L$. Par définition, $\gamma(\alpha)$ est la classe d'isomorphisme de $L$. 
1.7 - Étude de GL(p)-fibrés en droites sur $R^{s s}$. Considérons le foncteur

$$
F^{\prime} \text { : Variétés algébriques lisses } \longrightarrow \text { Ensembles }
$$

associant à $S$ l'ensemble des classes d'isomorphisme de familles de faisceaux de $M\left(r, c_{1}, c_{2}\right)$ paramétrées par $S$ (alors que $F\left(r, c_{1}, c_{2}\right)(S)$ est l'ensemble des classes d'équivalence de telles familles). On définit comme pour $F\left(r, c_{1}, c_{2}\right)$ le groupe de Picard $\operatorname{Pic}\left(F^{\prime}\right)$ de $F^{\prime}$, qui contient $\operatorname{Pic}\left(F\left(r, c_{1}, c_{2}\right)\right)$. On définit comme dans 1.6 des morphismes de groupes

$$
K(X) \stackrel{\gamma^{\prime}}{\longrightarrow} \operatorname{Pic}\left(F^{\prime}\right) \longrightarrow \operatorname{Pic}^{G^{\prime}}\left(R^{s s}\right),
$$

$\operatorname{Pic}^{G^{\prime}}\left(R^{s s}\right)$ désignant le groupe des classes d'isomorphisme de GL $(p)$-fibrés en droites sur $R^{s s}$. Pour tout $\alpha \in K(X)$ et toute famille $E$ de faisceaux de $M\left(r, c_{1}, c_{2}\right)$ paramétrée par une variété lisse $S$, on a

$$
\gamma^{\prime}(\alpha)_{E}=\operatorname{det}\left(p_{S !}\left([E] \otimes p_{X}^{*}(\alpha)\right)\right) .
$$

Le GL $(p)$-fibré en droites associé à $\alpha$ est donc

$$
L(\alpha)=\operatorname{det}\left(p_{R^{s s !}}\left(\mathbb{E} \otimes p_{X}^{*}(\alpha)\right)\right) .
$$

Pour tout $t \in \mathbb{C}^{*}$, l'action de $t$ sur une fibre de $L(\alpha)$ est la multiplication par $t^{m(\alpha)}$ (cf. 0.4).

Nous aurons besoin de savoir quels sont les $m(\alpha)$ possibles. On reprend les notations de 0.6 et 0.4 .

Lemme 1.4: L'image du morphisme $m: K(X) \rightarrow \mathbb{Z}$ est le saur-groupe $\mathbb{Z} d$.

Démonstration. On peut supposer que $H^{2}(X, \mathbb{Z})$ est de la forme

$$
H^{2}(X, \mathbb{Z}) \simeq H \oplus \mathbb{Z} E_{1} \oplus \cdots \oplus \mathbb{Z} E_{k},
$$

avec

$$
\begin{gathered}
E_{i}^{2}=-1, \quad E_{i} \cdot H=0 \quad \text { pour } 1 \leq i \leq k, \\
E_{i} \cdot E_{j}=0 \text { pour } 1 \leq i<j \leq k,
\end{gathered}
$$

$H$ étant le $H^{2}$ d'une surface rationnelle minimale.

Soient $r^{\prime}, \chi^{\prime}$ des entiers, $c_{1}^{\prime} \in H^{2}(X, \mathbb{Z})$. Alors, si $\alpha^{\prime} \in K(X)$ est de rang $r^{\prime}$, et si $c_{1}(\alpha)=c_{1}^{\prime}$, $\chi(\alpha)=\chi^{\prime}$, on a d'après le théorème de Riemann-Roch $m(\alpha)=r^{\prime} \chi+r\left(\chi^{\prime}-r^{\prime}\right)+c_{1} c_{1}^{\prime}$. Supposons que

$$
c_{1}=f+b_{1} E_{1}+\cdots+b_{k} E_{k}, \quad c_{1}^{\prime}=f^{\prime}+b_{1}^{\prime} E_{1}+\cdots+b_{k}^{\prime} E_{k},
$$

avec $f, f^{\prime} \in H, b_{i}, b_{i}^{\prime}$ entiers. Alors

$$
m(\alpha)=r^{\prime} \chi+r\left(\chi^{\prime}-r^{\prime}\right)+f \cdot f^{\prime}-b_{1} b_{1}^{\prime}-\cdots-b_{k} b_{k}^{\prime} .
$$

En considérant les différents cas possibles pour $H$, on voit aisément que les valeurs prises par $m$ sont les multiples de $d$.

Ceci démontre le lemme 1.4.

Lemme 1.5 : Tout $\mathrm{GL}(p)$-fibré en droites sur $R^{s}$ peut être prolongé en un $\mathrm{GL}(p)$-fibré en droites sur $R^{s s}$.

([7], lemme 5.2.) 


\section{CARACTÉRisation Des points faCtoriels}

2.1 - Démonstration des théorèmes A et B. On démontrera plus loin le

Lemme 2.1 : Soient $z, z^{\prime}$ des points fermés de $M\left(r, c_{1}, c_{2}\right)$, avec $z \geq z^{\prime}$. Soient $y, y^{\prime}$ des points de $R^{s s}$ au dessus de $z, z^{\prime}$ respectivement, tels que les orbites $\mathrm{PGL}(p) y$ et $\mathrm{PGL}(p) y^{\prime}$ soient fermées. On note $G_{y}, G_{y^{\prime}}$ les stabilisateurs de $y, y^{\prime}$. Soit $L$ un $\operatorname{PGL}(p)$-fibré en droites sur $R^{s s}$. Alors, si $G_{y^{\prime}}$ agit trivialement sur $L_{y^{\prime}}, G_{y}$ agit aussi trivialement sur $L_{y}$.

Il est clair que le théorème $\mathrm{A}$ entraine le théorème $\mathrm{B}$, compte tenu du lemme 2.1. Démontrons le théorème $\mathrm{A}$. Rappelons qu'on se place sous l'hypothèse où $M^{s}\left(r, c_{1}, c_{2}\right)$ est dense dans $M\left(r, c_{1}, c_{2}\right)$ et où la codimension du complémentaire est au moins 2.

D'après [15], p. 141, $\mathcal{O}_{z}$ est factoriel si et seulement si pour toute hypersurface $Y$ de $M\left(r, c_{1}, c_{2}\right)$, le faisceau d'idéaux $I_{Y}$ de $Y$ est libre en $z$. On va d'abord démontrer que les deux propriétés suivantes sont équivalentes :

(i) $\mathcal{O}_{z}$ est factoriel.

(ii) Pour tout fibré en droites $L_{0}$ sur $M^{s}\left(r, c_{1}, c_{2}\right)$, il existe un ouvert $U$ de $M\left(r, c_{1}, c_{2}\right)$ contenant $M^{s}\left(r, c_{1}, c_{2}\right)$ et $z$, tel que $L_{0}$ se prolonge en un fibré en droites sur $U$.

Supposons que (i) soit vérifiée. Démontrons (ii). On peut se limiter au cas où $L_{0}=I_{Y}, Y$ étant une hypersurface de $M^{s}\left(r, c_{1}, c_{2}\right)$. L'extension de $L_{0}$ est alors $I_{\bar{Y} \cap U}, \bar{Y}$ désignant l'adhérence de $Y$ dans $M\left(r, c_{1}, c_{2}\right)$ et $U$ l'ouvert de $M\left(r, c_{1}, c_{2}\right)$ où $I_{\bar{Y}}$ est localement libre.

Réciproquement, supposons (ii) vérifiée. Soit $Y$ une hypersurface de $M\left(r, c_{1}, c_{2}\right)$. Il faut montrer que $I_{Y}$ est libre en $z$. On applique (ii) à

$$
L_{0}=I_{Y \cap M^{s}\left(r, c_{1}, c_{2}\right)} \cdot
$$

On note $\bar{L}_{0}$ l'extension de $L_{0}$ à $U$. Le fibré $\bar{L}_{0}$ correspond à un diviseur $D$ de $U: \bar{L}_{0}=\mathcal{O}_{U}(D)$. Donc

$$
L_{0}=\mathcal{O}_{M^{s}\left(r, c_{1}, c_{2}\right)}\left(D \cap M^{s}\left(r, c_{1}, c_{2}\right)\right) .
$$

Les diviseurs $Y \cap M^{s}\left(r, c_{1}, c_{2}\right)$ et $-D \cap M^{s}\left(r, c_{1}, c_{2}\right)$ de $M^{s}\left(r, c_{1}, c_{2}\right)$ sont donc linéairement équivalents. Puisque $M^{s}\left(r, c_{1}, c_{2}\right)$ est dense dans $U$, que le complémentaire est de codimension au moins 2 , et que $M\left(r, c_{1}, c_{2}\right)$ est normale, on a $Y \cap U \equiv-D$. Donc $\bar{L}_{0}=I_{Y \mid U}$, et $I_{Y}$ est libre en $z$. L'équivalence de (i) et (ii) est donc prouvée.

On va maintenant montrer que (ii) est équivalente à

(iii) Pour tout PGL $(p)$-fibré en droites $L$ sur $R^{s s}$, le stabilisateur de $y$ agit trivialément sur $L_{y}$.

Le théorème A sera alors démontré.

Supposons (ii) vérifiée, et soit $L$ un $\mathrm{PGL}(p)$-fibré en droites sur $R^{s s}$. Alors $L_{\mid R^{s}}$ descend à $M^{s}\left(r, c_{1}, c_{2}\right)$. Soit $F=\left(L_{\mid R^{s}}\right) / \operatorname{PGL}(p)$. D'après (ii), $F$ se prolonge en un fibré en droites sur $U$, voisinage de $z$ contenant $M^{s}\left(r, c_{1}, c_{2}\right)$. Le PGL $(p)$-fibré en droites $\pi^{*}(F)$ sur $\pi^{-1}(U)$ comcide avec $L$ sur $R^{s}$, donc aussi sur $\pi^{-1}(U)$. Puisque $z \in U$, le stabilisateur de $y$ agit trivialement sur $L_{y}$. 
Réciproquement, supposons (iii) vraie. Soit $L_{0}$ un fibré en droites sur $M^{s}\left(r, c_{1}, c_{2}\right)$. Alors $\pi^{*}\left(L_{0}\right)$ est un $\operatorname{PGL}(p)$-fibré en droites sur $R^{s}$. D'après le lemme 1.4, ce fibré se prolonge en un $\operatorname{PGL}(p)$ fibré en droites $L$ sur $R^{s s}$. Soit $Z$ l'ensemble des points $w$ de $R^{s s}$ tels que PGL $(p) w$ soit fermée, et que le stabilisateur de $w$ agisse trivialement sur $L_{w}$. Montrons que $\pi(Z)$ est un ouvert de $M\left(r, c_{1}, c_{2}\right)$. Si $z_{0} \in \pi(Z)$, alors d'après le lemme $2.1, \pi(Z)$ contient aussi tous les points $z^{\prime} \geq z_{0}$. Il suffit donc de démontrer le

Lemme 2.2 : Soit $z_{0}$ un point fermé de $M\left(r, c_{1}, c_{2}\right)$. Alors l'ensemble des points fermés $z_{1}$ de $M\left(r, c_{1}, c_{2}\right)$ tels que $z_{1} \geq z_{0}$ est un ouvert de $M\left(r, c_{1}, c_{2}\right)$.

Démonstration. On utilise les notations de 0.2.1. Soit $Z_{0} \subset M\left(r, c_{1}, z_{2}\right)$ l'ensemble des $z \in M\left(r, c_{1}, c_{2}\right)$ qui ne sont pas $\geq z_{0}$. Si $z \in Z_{0}$, d'après $0.2 .1, Z$ contient une sous-variété fermée du type $\alpha(\bar{U})$ contenant $Z_{0}$. Comme ces sous-veriétés sont en nombre fini, $Z_{0}$ est fermé. Ceci prouve le lemme 2.2 .

On pose $U=\pi^{-1}(\pi(Z))$. C'est un ouvert de $R^{s s}$, contenant $y$. D'après le lemme de descente (thm. 1.1), $L_{\mid U}$ descend à $\pi(Z)$, c'est à dire qu'il existe un fibré en droites $L^{\prime}$ sur $\pi(Z)$ et un $\operatorname{PGL}(p)$-isomorphisme $L_{\mid U} \simeq \pi^{*}\left(L^{\prime}\right)$. L'ouvert $\pi(Z)$ est un voisinage de $z$ contenant $M^{s}\left(r, c_{1}, c_{2}\right)$ et $L^{\prime}$ est l'extension cherchée de $L_{0}$. Ceci prouve (iii).

Ceci achève la démonstration du théorème $\mathrm{A}$.

2.2 - Démonstration du lemme 2.1. Elle s'inspire de celle de la proposition 4.1 de [7]. Comme dans [7], l'orbite $\operatorname{PGL}(p) y$ est fermée si et seulement si $\mathbb{E}_{y}$ est isomorphe à son gradué. On peut donc écrire

$$
\mathbb{E}_{y}=m_{1} E_{1} \oplus \cdots \oplus m_{k} E_{k}
$$

les $E_{i}$ étant des faisceaux stables, non isomorphes deux à deux, les $m_{i}$ des entiers $\geq 1$. Le stabilisateur de $y$ dans $\mathrm{GL}(p)$ s'identifie à

$$
\operatorname{Aut}\left(\mathbb{E}_{y}\right)=\operatorname{GL}\left(m_{1}\right) \times \cdots \times \operatorname{GL}\left(m_{k}\right) .
$$

On a donc

$$
G_{y}=\left(\mathrm{GL}\left(m_{1}\right) \times \cdots \times \mathrm{GL}\left(m_{k}\right)\right) / \mathbb{C}^{*} .
$$

L'action de $G_{y}$ sur $L_{y}$ est de la forme

$$
\begin{aligned}
\mathbb{G} \times L_{y} \longrightarrow L_{y} \\
(g, u) \longmapsto \lambda(g) u,
\end{aligned}
$$

$\lambda$ étant un caractère de $G_{y}$.

Il est clair que dans la démonstration du lemme 2.1 on peut remplacer $y^{\prime}$ par n'importe quel point de son orbite. La condition $z^{\prime} \leq z$ signifie qu'on peut écrire

$$
\mathbb{E}_{y^{\prime}} \simeq m_{1} E_{1}^{\prime} \oplus \cdots \oplus m_{k} E_{k}^{\prime},
$$


où pour $1 \leq i \leq k, E_{i}^{\prime}$ est un faisceau semi-stable dont la classe de $S$-équivalence est dans la variété de modules contenant la classe d'isomorphisme de $E_{i}$, et même dans la même composante irréductible. Quitte à remplacer $y^{\prime}$ par un élément de son orbite, on peut supposer que l'isomorphisme

$$
\bigoplus_{1 \leq i \leq k} H^{0}\left(E_{i}(m)\right) \otimes \mathbb{C}^{m_{i}} \simeq \bigoplus_{1 \leq i \leq k} H^{0}\left(E_{i}^{\prime}(m)\right) \otimes \mathbb{C}^{m_{i}}
$$

obtenu en composant les isomorphismes

$$
\bigoplus_{1 \leq i \leq k} H^{0}\left(E_{i}(m)\right) \otimes \mathbb{C}^{m_{i}} \simeq H^{0}\left(\mathbb{E}_{y}(m)\right) \stackrel{H^{0}\left(\theta_{y}\right)^{-1}}{\longrightarrow} \mathbb{C}^{p}
$$

et

$$
\mathbb{C}^{p} \stackrel{H^{0}\left(\theta_{y^{\prime}}\right)}{\longrightarrow} H^{0}\left(\mathbb{E}_{y}^{\prime}(m)\right) \simeq \bigoplus_{1 \leq i \leq k} H^{0}\left(E_{i}^{\prime}(m)\right) \otimes \mathbb{C}^{m_{i}}
$$

envoie $H^{0}\left(E_{i}(m)\right) \otimes \mathbb{C}^{m_{i}}$ sur $H^{0}\left(E_{i}^{\prime}(m)\right) \otimes \mathbb{C}^{m_{i}}$ pour $1 \leq i \leq k$, et que l'isomorphisme induit

$$
H^{0}\left(E_{i}(m)\right) \otimes \mathbb{C}^{m_{i}} \simeq H^{0}\left(E_{i}^{\prime}(m)\right) \otimes \mathbb{C}^{m_{i}}
$$

est de la forme $\sigma_{i} \otimes I d, \sigma_{i}$ étant un isomorphisme $H^{0}\left(E_{i}(m)\right) \simeq H^{0}\left(E_{i}^{\prime}(m)\right)$. Il suffit de prouver le

Lemme 2.3 : Il existe une variété algébrique intègre $R_{0}$, et un morphisme $\phi: R_{0} \rightarrow R^{s s}$ tels que :

(i) L'image de $\phi$ contient $y$ et $y^{\prime}$.

(ii) Pour tout point $y_{0}$ de l'image de $\phi$, le stabilisateur $G_{y_{0}}$ de $y_{0}$ contient $G_{y}$.

En effet, supposons le lemme 2.3 démontré. Alors les propriétés (i) et (ii) sont encore vérifiées si on remplace l'image de $\phi$ par son adhérence $S$ qui est une sous-variété irréductible de $R^{s s}$ sur laquelle $G_{y}$ agit trivialement. L'action de $G_{y}$ sur $L_{\mid S}$ s'écrit

$$
\begin{aligned}
G_{y} \times L_{\mid S} \longrightarrow L_{\mid S} \\
(g, u) \longmapsto \lambda_{\alpha(u)}(g) u,
\end{aligned}
$$

où $\alpha: L_{\mid S} \rightarrow S$ est la projection, et pour tout $s \in S, \lambda_{s}$ est un caractère de $G_{y}$.

Montrons que pour tout $s \in S$, on a $\lambda_{s}=\lambda$. Soit $g \in G_{y}$. En prenant des trivialisations locales de $L_{\mid S}$, on voit que

$$
\begin{aligned}
G_{y} \times L_{\mid S} & \longrightarrow \mathbb{C}^{*} \\
(g, u) & \longmapsto \lambda_{\alpha(u)}(g)
\end{aligned}
$$

est un morphisme. Puisque le groupe des caractères de $G_{y}$ est dénombrable, pour tout $g \in G_{y}$, le morphisme

$$
\begin{aligned}
& L_{\mid S} \longrightarrow \mathbb{C}^{*} \\
& u \longmapsto \lambda_{\alpha(u)}(g)
\end{aligned}
$$

prend une quantité dénombrable de valeurs, donc est constant. Le caractère $\lambda_{s}$ ne dépend donc pas du point $s$ de $S$. Donc $\lambda_{s}=\lambda_{y}=\lambda$. 
On a $G_{y} \subset G_{y^{\prime}}$, et $G_{y^{\prime}}$ agit trivialement sur $L_{y^{\prime}}$, donc $G_{y}$ aussi. D'où $\lambda_{y^{\prime}}=1$, donc $\lambda=1$, ce qui prouve le lemme 2.1. ll reste à démontrer le lemme 2.3.

Un morphisme $\phi: R_{0} \rightarrow R^{s s}$ équivaut à la donnée d'un morphisme surjectif de faisceaux sur $R_{0} \times X$ :

$$
p_{X}^{*}\left(\mathcal{O}_{X}(-m)\right) \otimes \mathbb{C}^{p} \stackrel{\theta^{\prime}}{\longrightarrow} \mathbb{F}
$$

où $\mathbb{F}$ est une famille de faisceaux de $M\left(r, c_{1}, c_{2}\right)$ paramétrée par $R_{0}$, tel qu'en tout point fermé $x$ de $R_{0}, \theta_{x}^{\prime}$ induise un isomorphisme $\mathbb{C}^{p} \rightarrow H^{0}\left(\mathbb{F}_{x}((m))\right.$. La condition (ii) équivaut à la suivante : pour tout $g \in G_{y}$ on a un diagramme commutatif

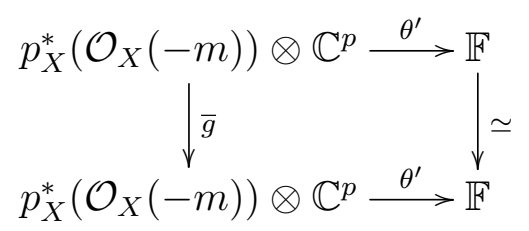

$(\bar{g}$ étant un élément de $\mathrm{GL}(p)$ au desus de $g)$.

On considère, pour $1 \leq i \leq k$, le schéma de Grothendieck $R_{i}^{s s}$ correspondant à $E_{i}$,

$$
\theta_{i}: p_{X}^{*}\left(\mathcal{O}_{X}(-m)\right) \otimes \mathbb{C}^{p_{i}} \longrightarrow \mathbb{E}_{i}
$$

le morphisme surjectif universel sur $R_{i}^{s s} \times X$. On note $W_{i}$ la composante irréductible de $R_{i}^{s s}$ contenant un point $x$ tel que $\mathbb{E}_{i x} \simeq E_{i}$. La condition $z \geq z^{\prime}$ implique que $W_{i}$ contient un point $x^{\prime}$ tel que $\mathbb{E}_{i x^{\prime}} \simeq E_{i}^{\prime}$.

Fixons des isomorphismes $\mathbb{C}^{p_{i}} \simeq H^{0}\left(E_{i}(m)\right)$ pour $1 \leq i \leq k$. Alors on a un isomorphisme

$$
\mathbb{C}^{p} \simeq \bigoplus_{1 \leq i \leq k}\left(\mathbb{C}^{p_{i}} \otimes \mathbb{C}^{m_{i}}\right)
$$

obtenu en prenant le composé

$$
\mathbb{C}^{p} \stackrel{\theta_{y}}{\longrightarrow} H^{0}\left(\mathbb{E}_{y}(m)\right) \simeq H^{0}\left(\bigoplus_{1 \leq i \leq k} E_{i}(m) \otimes \mathbb{C}^{m_{i}}\right) \simeq \bigoplus_{1 \leq i \leq k} H^{0}\left(E_{i}(m)\right) \otimes \mathbb{C}^{m_{i}}=\bigoplus_{1 \leq i \leq k}\left(\mathbb{C}^{p_{i}} \otimes \mathbb{C}^{m_{i}}\right) .
$$

On prend maintenant $R_{0}=W_{1} \times \cdots \times W_{k}$, et $\theta^{\prime}$ est le composé

$$
\mathbb{C}^{p} \otimes p_{X}^{*}\left(\mathcal{O}_{X}(-m)\right) \simeq\left(\bigoplus_{1 \leq i \leq k}\left(\mathbb{C}^{p_{i}} \otimes \mathbb{C}^{m_{i}}\right)\right) \otimes p_{X}^{*}\left(\mathcal{O}_{X}(-m)\right) \stackrel{\beta}{\longrightarrow} \bigoplus_{1 \leq i \leq k} p_{W_{i}}^{\#}\left(\mathbb{E}_{i}\right) \otimes \mathbb{C}^{m_{i}}
$$

avec

$$
\beta=\bigoplus_{1 \leq i \leq k}\left(p_{W_{i}}^{\#}\left(\theta_{i}\right) \otimes I d\right)
$$


Il est immédiat que la condition (i) est satisfaite. Vérifions (ii). Cela découle du diagramme commutatif suivant, pour tout $g=\left(g_{1}, \ldots, g_{k}\right) \in \mathrm{GL}\left(m_{1}\right) \times \ldots \times \mathrm{GL}\left(m_{k}\right)$

$$
\begin{aligned}
& \mathbb{C}^{p} \otimes p_{X}^{*}\left(\mathcal{O}_{X}(-m)\right) \simeq\left(\bigoplus_{1 \leq i \leq k}\left(\mathbb{C}^{p_{i}} \otimes \mathbb{C}^{m_{i}}\right)\right) \otimes p_{X}^{*}\left(\mathcal{O}_{X}(-m)\right) \stackrel{\beta}{\longrightarrow} \underset{1 \leq i \leq k}{\bigoplus p_{W_{i}}^{\#}\left(\mathbb{E}_{i}\right) \otimes \mathbb{C}^{m_{i}}} \\
& \mathbb{C}^{p} \otimes p_{X}^{*}\left(\mathcal{O}_{X}(-m)\right) \simeq\left(\bigoplus_{1 \leq i \leq k}\left(\mathbb{C}^{p_{i}} \otimes \mathbb{C}^{m_{i}}\right)\right) \otimes p_{X}^{*}\left(\mathcal{O}_{X}(-m)\right) \stackrel{\beta}{\downarrow} \longrightarrow \bigoplus_{1 \leq i \leq k} \bigoplus_{W_{i}} p_{W_{i}}^{\#}\left(\mathbb{E}_{i}\right) \otimes \mathbb{C}^{m_{i}}
\end{aligned}
$$

Ceci achève la démonstration du lemme 2.1.

\section{NON-FACTORIALité DES POINTS DE TYPE 2}

On démontre ici le théorème C. On a défini dans 1.6 un morphisme de groupes

$$
\gamma: H\left(r, c_{1}, c_{2}\right) \longrightarrow \operatorname{Pic}^{G}\left(R^{s s}\right) \text {. }
$$

Soit $\alpha \in H\left(r, c_{1}, c_{2}\right)$. On peut écrire

$$
\alpha=\sum_{1 \leq i \leq q} n_{i}\left[F_{i}\right],
$$

les $F_{i}$ étant des fibrés vectoriels sur $X$, et les $n_{i}$ des entiers. Soit $y \in R^{s s}$. Soit $G_{y}^{\prime}$ le stabilisateur de $y$ dans $\mathrm{GL}(p), G_{y}=G_{y}^{\prime} / \mathbb{C}^{*}$ son stabilisateur dans $\operatorname{PGL}(p)$. On sait que $G_{y}^{\prime}$ sidentifie à $\operatorname{Aut}\left(\mathbb{E}_{y}\right)$. L'action de $\operatorname{Aut}\left(\mathbb{E}_{y}\right)$ sur $\gamma(\alpha)_{y}$ est équivalente à son action sur

$$
L=\bigotimes_{0 \leq j \leq 2}\left(\bigotimes_{1 \leq i \leq q} \operatorname{det}\left(H^{j}\left(\mathbb{E}_{y} \otimes F_{i}\right)^{\otimes n_{i}}\right)\right) \text {. }
$$

Supposons que PGL $(p) y$ soit fermée et que $\pi(y)=z$ soit de type 2 . Pour démontrer le théorème $\mathrm{C}$, il suffit de prouver qu'il existe un $\alpha$ dans $H\left(r, c_{1}, c_{2}\right)$ tel que l'action de $\operatorname{Aut}\left(\mathbb{E}_{y}\right)$ sur $L$ ne soit pas triviale. On peut écrire

$$
\mathbb{E}_{y}=m_{1} E_{1} \oplus \cdots \oplus m_{k} E_{k}
$$

les $E_{i}$ étant des faisceaux stables non isomorphes deux à deux, les $m_{i}$ des entiers $\geq 1$. On a alors $G_{y}^{\prime} \simeq \mathrm{GL}\left(m_{1}\right) \times \cdots \mathrm{GL}\left(m_{k}\right)$. L'action de $G_{y}^{\prime}$ sur $\gamma(\alpha)_{y}=L$ est définie par le caractère

$$
\begin{aligned}
\lambda: \operatorname{GL}\left(m_{1}\right) \times \cdots \mathrm{GL}\left(m_{k}\right) & \longrightarrow \mathbb{C}^{*} \\
\left(g_{1}, \ldots, g_{k}\right) & \longmapsto \prod_{1 \leq i \leq k} \operatorname{det}\left(g_{i}\right)^{\chi\left(\left[E_{i}\right] \otimes \alpha\right)} .
\end{aligned}
$$

Il suffit donc de montrer qu'il existe un $\alpha$ dans $H\left(r, c_{1}, c_{2}\right)$ et un $i$ tels que $\chi\left(\left[E_{i}\right] \otimes \alpha\right) \neq 0$. Pour tout faisceau cohérent $F$ sur $X$, de rang non nul, on pose $\mu(F)=\frac{c_{1}(F)}{\operatorname{rg}(F)} \in H^{2}(X, \mathbb{Q})$. 
Puisque $z$ est de type 2, il existe un $i$ tel que $\mu\left(E_{i}\right) \neq \frac{c_{1}}{r}$. Il reste à montrer que les formes linéaires sur $K(X)$

$$
\phi_{1}: \alpha \longrightarrow \chi\left(\left[E_{i}\right] \otimes \alpha\right), \quad \phi_{2}: \alpha \longrightarrow \chi\left(\left[\mathbb{E}_{y}\right] \otimes \alpha\right)
$$

ne sont pas proportionnelles. Supposons le contraire. Il existe alors des entiers $a, b$ non tous nuls tels que pour tout $\alpha \in K(X)$ on ait

$$
\chi\left(\left(a\left[\mathbb{E}_{y}\right]-b\left[E_{i}\right]\right) \otimes \alpha\right)=0 .
$$

Supposons que

$$
\begin{aligned}
r_{0} & =\operatorname{rg}\left(a\left[\mathbb{E}_{y}\right]-b\left[E_{i}\right]\right), \\
x_{0} & =c_{1}\left(a\left[\mathbb{E}_{y}\right]-b\left[E_{i}\right]\right), \\
y_{0} & =c_{2}\left(a\left[\mathbb{E}_{y}\right]-b\left[E_{i}\right]\right), \\
R & =\operatorname{rg}(\alpha), \\
X & =c_{1}(\alpha), \\
Z & =c_{2}(\alpha),
\end{aligned}
$$

Alors on a

$$
R\left(x_{0}^{2}-K x_{0}+2 r_{0}-2 y_{0}\right)+r_{0} X^{2}+\left(2 x_{0}-r_{0} K\right) X-2 r_{0} Y=0,
$$

pour tous entiers $R, Y$ et tout $X \in A^{1}(X)$. On en déduit déjà que $r_{0}=0$ (le coefficient de l'unique terme contenant $Y$ est nul). On a donc

$$
R\left(x_{0}^{2}-K x_{0}+2 y_{0}\right)+2 x_{0} X=0 .
$$

Puisque la forme d'intersection sur $A^{1}(X)$ est non dégénérée, on a $x_{0}=0$. Donc $y_{0}=0$. Finalement on obtient d'après 1.5

$$
a\left[\mathbb{E}_{y}\right]-b\left[E_{i}\right]=0 .
$$

Mais ceci entraine $\mu\left(E_{i}\right)=\frac{c_{1}}{r}$, contrairement à l'hypothèse. Donc $\phi_{1}$ et $\phi_{2}$ ne sont pas proportionnelles,

Ceci achève la démonstration du théorème C.

\section{Faisceaux universels}

On démontre ici le théorème $\mathrm{D}$. On reprend les notations de 0.6. La démonstration de (1) est exactement la même que celle du théorème $\mathrm{G}$ de [7]. On se contentera de l'esquisser. On montre d'abord comme dans l'article cité qu'il existe un faisceau de Poincaré sur $U$ si et seulement si il en existe un sur $M^{s}\left(r, c_{1}, c_{2}\right)$ (rappelons qu'ici $M\left(r, c_{1}, c_{2}\right)$ est supposée irréductible). On considère l'action de $\mathrm{GL}(p)$ sur $\mathbb{E}$. Pour tout $y \in R^{s s}$ et tout $t \in \mathbb{C}^{*} \subset \mathrm{GL}(p)$, l'action de $t$ sur $\mathbb{E}_{y}$ est la multiplication par $t$. Il en découle que l'existence d'un faisceau de Poincaré sur $M^{s}\left(r, c_{1}, c_{2}\right)$ équivaut à celle d'un $\mathrm{GL}(p)$-fibré en droites $L$ sur $R^{s}$ tel que pour tout $y \in R^{s}$ et tout $t \in \mathbb{C}^{*}$, l'action de $t$ sur $L_{y}$ soit la multiplication par $t$. En effet, si un tel $L$ existe, on prend 
pour faisceau de Poincaré sur $M^{s}\left(r, c_{1}, c_{2}\right)$ le faisceau $\left(E \otimes L^{-1}\right) / \operatorname{PGL}(p)$, et réciproquement, si $F$ est un faisceau de Poincaré sur $M^{s}\left(r, c_{1}, c_{2}\right)$, on prend $L=p_{R^{s} *}\left(\mathcal{H o m}\left(\pi^{*}(F), \mathbb{E}\right)\right)$.

Soit $L^{\prime}$ un GL $(p)$-fibré en droites sur $R^{s s}$. Il existe alors un entier $k$ tel que pour tout $y \in R^{s s}$ et tout $t \in \mathbb{C}^{*}$, l'action de $t$ sur $L_{y}^{\prime}$ soit la multiplication par $t^{k}$. On note alors $e\left(L^{\prime}\right)=k$.

Soit $L$ un $\operatorname{GL}(p)$-fibré en droites sur $R^{s}$. D'aprés le lemme 1.5, on peut prolonger $L$ en un $\operatorname{GL}(p)$-fibré en droites $\bar{L}$ sur $R^{s s}$. Pour démontrer (1), il suffit de prouver l'assertion suivante : pour tout $\mathrm{GL}(p)$-fibré en droites $L^{\prime}$ sur $R^{s s}$, on a $e\left(L^{\prime}\right) \neq 1$.

Pour cela, on considère un point fermé $y$ de $R^{s s}$ tel que $\operatorname{PGL}(p) y$ soit fermée et que $\pi(y)$ soit spécial. Alors on a $\mathbb{E}_{y} \simeq n E$, avec $n>1$ et $E$ stable. Donc le stabilisateur de $y$ dans $\operatorname{GL}(p)$ s'identifie à $\mathrm{GL}(n)$. L'action de $\mathrm{GL}(n)$ sur $L_{y}$ est de la forme

$$
\begin{aligned}
\operatorname{GL}(n) \times L_{y} & \longrightarrow L_{y} \\
(g, u) & \longmapsto \operatorname{det}(g)^{q} u
\end{aligned}
$$

avec $q$ entier. Il en découle que $e\left(L^{\prime}\right)=q n$, et ne peut donc pas être égal à 1. Ceci prouve (1). Démontrons maintenant (2). On a défini dans 1.7 un morphisme de groupes

$L: K(X) \rightarrow \operatorname{Pic}^{G^{\prime}}\left(R^{s s}\right)$. On avu dans 1.7 que pour tout $\alpha \in K(X)$ on a $e(L(\alpha))=m(\alpha)$. D'après le lemme 1.4, sous les hypothèses de (2) il existe un $\alpha \in K(X)$ tel que $m(\alpha)=1$. On a donc $e(L(\alpha))=1$, ce qui entraine comme on l'a vu l'existence d'un faisceau de Poincaré sur $M^{s}\left(r, c_{1}, c_{2}\right)$.

Ceci achève la démonstration du théorème D.

\section{5. Étude D'un CAS PARTiCUlier}

On s'intéresse au cas suivant : $X=\mathbb{P}_{1} \times \mathbb{P}_{1}, r=2, c_{1}=0$. Supposons que $\mathcal{O}_{X}(1)=\mathcal{O}(\alpha, \beta)$, $\alpha$ et $\beta$ étant deux entiers positifs premiers entre eux. On a $K=\mathcal{O}(-2,-2)$, donc $K . \mathcal{O}_{X}(1)=-2(\alpha+\beta)<0$.

Soit $E$ un faisceau semi-stable sur $X$, de rang 2 et de classes de Chern $0, c_{2}$. D'après [13], lemma 2.4, si $c_{2} \leq 0$, on a $E \simeq 2 \mathcal{O}$. On supposera donc que $c_{2}>0$. La semi-stabilité de $E$ entraine que $h^{0}(E)=0$. Puisque $K . \mathcal{O}_{X}(1)<0$, on a aussi

$$
h^{2}(E)=\operatorname{dim}(\operatorname{Hom}(E, K))=0,
$$

donc $\chi(E) \leq 0$. La formule de Riemann-Roch donne $\chi(E)=2-c_{2}$. On a donc $c_{2} \geq 2$.

5.1 - Monades. Ce qui suit servira essentiellement à prouver l'irréductibilité de $M\left(2,0, c_{2}\right)$. On note $p_{1}, p_{2}$ les projections $X \times X \rightarrow X$. Si $E, F$ sont des faisceaux cohérents sur $X$, on notera

$$
E \otimes F=p_{1}^{*}(E) \otimes p_{2}^{*}(F)
$$


Soit $\Delta$ la diagonale de $X \times X$. On a alors une résolution de $\mathcal{O}_{\Delta}$ :

$$
0 \longrightarrow \mathcal{O}(-1,-1) \otimes \mathcal{O}(-1,-1) \stackrel{u}{\longrightarrow}\left[\begin{array}{c}
\mathcal{O}(-1,0) \underset{\mathcal{O}(-1,0)}{\oplus} \\
\mathcal{O}(0,-1) \otimes \mathcal{O}(0,-1)
\end{array}\right] \stackrel{v}{\longrightarrow} \mathcal{O} \longrightarrow \mathcal{O}_{\Delta} \longrightarrow 0
$$

Pour définir cette résolution, posons $\mathbb{P}_{1}=\mathbb{P}(D)$ (droites de $D$ ), $D$ étant un $\mathbb{C}$-espace vectoriel de dimension 2. On fixe un isomorphisme $\wedge^{2} D \simeq \mathbb{C}$. On définit $v$ et $u$ par

$$
\begin{aligned}
& v_{(x, y),\left(x^{\prime}, y^{\prime}\right)}\left(X \otimes X^{\prime}+Y \otimes Y^{\prime}\right)=X \wedge X^{\prime}+Y \wedge Y^{\prime} \\
& u_{(x, y),\left(x^{\prime}, y^{\prime}\right)}\left(X \otimes Y \otimes X^{\prime} \otimes Y^{\prime}\right)=\left(Y \wedge Y^{\prime}\right) X \otimes X^{\prime}-\left(X \wedge X^{\prime}\right) Y \otimes Y^{\prime},
\end{aligned}
$$

pour tous points $(x, y),\left(x^{\prime}, y^{\prime}\right)$ de $\mathbb{P}_{1} \times \mathbb{P}_{1}$, et tous $X, Y, X^{\prime}, Y^{\prime}$ dans $x, y, x^{\prime}, y^{\prime}$ respectivement (vus comme des droites de $D$ ).

Il est aisé de vérifier qu'on obtient ainsi une résolution de $\mathcal{O}_{\Delta}$. On en déduit comme dans le cas de $\mathbb{P}_{2}$ une "suite spectrale de Beilinson" sur $\mathbb{P}_{1} \times \mathbb{P}_{1}$ (cf. [2]) :

Proposition 5.1 : Soit $E$ un faisceau cohérent sur $\mathbb{P}_{1} \times \mathbb{P}_{1}$. Alors il existe une suite spectrale $E_{r}^{p, q}$ de faisceaux cohérents sur $\mathbb{P}_{1} \times \mathbb{P}_{1}$, convergeant vers $E$ en degré 0 , vers 0 en tout autre degré, dant les termes $E_{1}^{p, q}$ éventuellement non nuls sont:

$$
\begin{aligned}
E_{1}^{-2, i} & =H^{i}(E(-1,-1)) \otimes \mathcal{O}(-1,-1), \\
E_{1}^{-1, i} & =\left(H^{i}(E(-1,0)) \otimes \mathcal{O}(-1.0)\right) \oplus\left(H^{i}(E(0,-1)) \otimes \mathcal{O}(0-1)\right), \\
E_{1}^{0, i} & =H^{i}(E) \otimes \mathcal{O},
\end{aligned}
$$

pour $i=0,1,2$.

Corollaire 5.2 : Soit E un faisceau cohérent sur $\mathbb{P}_{1} \times \mathbb{P}_{1}$, tel que

$$
h^{0}(E)=h^{2}(E(-1,-1))=0 .
$$

Alors il existe un complexe

$$
H^{1}(E(-1,-1)) \otimes \mathcal{O}(-1,-1) \stackrel{A}{\longrightarrow}\left[\begin{array}{c}
H^{1}(E(-1,0)) \otimes \mathcal{O}(-1,0) \\
\oplus \\
H^{1}(E(0,-1)) \otimes \mathcal{O}(0,-1)
\end{array}\right] \stackrel{B}{\longrightarrow} H^{1}(E) \otimes \mathcal{O}
$$

avec $A$ injectif (comme morphisme de faisceaux), $B$ surjectif, tel que $\operatorname{ker}(B) / \operatorname{im}(A) \simeq E$.

Un tel complexe s'appelle une monade (cf. [11]). On va utiliser le résultat précédent pour étudier $M\left(2,0, c_{2}\right)$ de la même façon que les monades sur $\mathbb{P}_{2}$ sont utilisées dans [6].

Soit $E$ un faisceau semi-stable de rang 2 et de classes de Chern $0, c_{2}=n \geq 2$. D'après le théorème de Riemann-Roch, on a

$$
\chi(E(-1,-1))=\chi(E(-1,0))=\chi(E(0,-1))=-n,
$$

donc $E$ est isomorphe à la oohomologie d'une monade du type

$$
\mathcal{O}(-1,-1) \otimes \mathbb{C}^{n} \stackrel{A}{\longrightarrow}(\mathcal{O}(-1,0) \oplus \mathcal{O}(0,-1)) \otimes \mathbb{C}^{n} \stackrel{B}{\longrightarrow} \mathcal{O} \otimes \mathbb{C}^{n-2},
$$

avec $A$ injectif comme morphisme de faisceaux et $B$ surjectif. Soit $\mathcal{M}$ la variété algébrique de tous les complexes de ce type. Si $(A, B)$ en est un, on notera $F_{(A, B)}=\operatorname{ker}(B) / \operatorname{im}(A)$. On 
montre que si $\operatorname{Ext}^{2}\left(F_{(A, B)}, F_{(A, B)}\right)=0$, alors $\mathcal{M}$ est lisse au point $(A, B)$ (cf. [6], prop. (2.6)). Soit $\mathcal{M}^{s s}$ l'ouvert de $\mathcal{M}$ des points $(A, B)$ tels que $F_{(A, B)}$ soit semi-stable. C'est un ouvert lisse de $\mathcal{M}$, car par dualité de Serre on a

$$
\operatorname{Ext}^{2}\left(F_{(A, B)}, F_{(A, B)}\right) \simeq \operatorname{Hom}\left(F_{(A, B)}, F_{(A, B)} \otimes K\right)=0
$$

par semi-stabilité de $F_{(A, B)}$. Sur $\mathcal{M}^{s s} \times X$, existe une monade universelle, dont la cohomologie est évidemment notée $F$. C'est donc une famille de faisceaux de $M\left(2,0, c_{2}\right)$ paramétrée par $\mathcal{M}^{s s}$.

Sur $\mathcal{M}^{s s}$ agit de manière évidente le groupe réductif

$$
G=\left(\operatorname{GL}(n)^{2} \times \operatorname{GL}(n-2)\right) / \mathbb{C}^{*}
$$

de telle sorte que le morphisme canonique

$$
f_{F}: \mathcal{M}^{s s} \longrightarrow M\left(2,0, c_{2}\right)
$$

soit un bon quotient de $\mathcal{M}^{s s}$ par $G$ (cf. [6], prop. (2.6)). La variété $\mathcal{M}^{s s}$ est équidimensionnelle, et on a $\operatorname{dim}\left(\mathcal{M}^{s s}\right)=4 c_{2}-3+\operatorname{dim}(G)$.

5.2 - Faisceaux semi-stables non stables. Soit $E$ un faisceau semi-stable non stable, de rang 2 et de classes de Chern $0, c_{2}$. On a une filtration de Jordan-Hölder $0 \subset F \subset E$, avec $\operatorname{rg}(F)=1, c_{1}(F) \cdot \mathcal{O}(\alpha, \beta)=0$ et $\chi(F)=1-\frac{c_{2}}{2}$. En particulier $c_{2}$ doit être pair pour qu'il existe de tels E. On supposera toujours que $c_{2}$ est pair dans la suite.

Le faisceau $F$ est de la forme $F=I_{Y}(a, b), Y$ étant un sous-schéma fini de $\mathbb{P}_{1} \times \mathbb{P}_{1}, a, b$, des entiers. La condition $c_{1}(F) \cdot(\alpha, \beta)=0$ implique que $a$ et $b$ sont de la forme

$$
a=k \alpha, \quad b=-k \beta,
$$

avec $k$ entier. On a une suite exacte

$$
0 \longrightarrow I_{Y}(a, b) \longrightarrow \mathcal{O}(a, b) \longrightarrow \mathcal{O}_{Y} \longrightarrow 0
$$

d'où

$$
\chi\left(I_{Y}(a, b)\right)=\chi(\mathcal{O}(a, b))-l(Y)=(1+k \alpha)(1-k \beta)-l(Y) .
$$

Mais $\chi\left(I_{Y}(a, b)\right)=1-\frac{c_{2}}{2}$, donc

$$
l(Y)=-k^{2} \alpha \beta+k(\alpha-\beta)+\frac{c_{2}}{2} .
$$

On montre de même que $E / F \simeq I_{Y^{\prime}}(-k \alpha, k \beta)$, où $Y^{\prime}$ est un sous-schéma fini de $\mathbb{P}_{1} \times \mathbb{P}_{1}$ de longueur

$$
l\left(Y^{\prime}\right)=-k^{2} \alpha \beta+k(\beta-\alpha)+\frac{c_{2}}{2} .
$$

Les conditions $l(Y) \geq 0, l\left(Y^{\prime}\right) \geq 0$ sont équivalentes à $|k| \leq m$, avec

$$
m=\left[\frac{-|\beta-\alpha|+\sqrt{(\alpha-\beta)^{2}+2 \alpha \beta c_{2}}}{2 \alpha \beta}\right] .
$$


Pour tout entier $c \geq 0$, on note $\operatorname{Hilb}^{c}(X)$ le schéma de Hilbert des sous-schémas finis de longueur $c$ de $X$. C'est une variété algébrique lisse et irréductible de dimension $2 c$. Pour tout entier $k$ tel que $0 \leq k \leq m$, on pose

$$
c_{k}=-k^{2} \alpha \beta+k(\alpha-\beta)+\frac{c_{2}}{2}, \quad d_{k}=-k^{2} \alpha \beta+k(\beta-\alpha)+\frac{c_{2}}{2},
$$

et on considère le morphisme

$$
\begin{aligned}
\Phi_{k}: \operatorname{Hilb}^{c_{k}}(X) \times \operatorname{Hilb}^{d_{k}}(X) & \longrightarrow M\left(2,0, c_{2}\right) \\
\left(Y, Y^{\prime}\right) \longmapsto & \longrightarrow\left[I_{Y}(k \alpha,-k \beta) \oplus I_{Y^{\prime}}(-k \alpha, k \beta)\right] .
\end{aligned}
$$

L'image $Z_{k}$ de $\Phi_{k}$ est une sous-variété fermée de $M\left(2,0, c_{2}\right)$ de dimension $2\left(c_{k}+d_{k}\right)=2\left(c_{2}-2 k^{2} \alpha \beta\right)$. Les $Z_{k}$ sont disjointes deux à deux et on a

$$
M\left(2,0, c_{2}\right) \backslash M^{s}\left(2,0, c_{2}\right)=Z_{0} \cup Z_{1} \cup \cdots \cup Z_{m} .
$$

La composante $Z_{0}$ est du même type que ce qu'on observe sur $\mathbb{P}_{2}$. Mais dès que $c_{2} \geq 2(\alpha \beta+|\alpha-\beta|)$, d'autres composantes apparaissent.

\section{3 - Irréductibilité de $M\left(2,0, c_{2}\right)$}

Proposition 5.3 : La variété $M\left(2,0, c_{2}\right)$ est irréductible.

Démonstration. Soit $M^{0 s}$ l'ouvert de $M^{s}\left(2,0, c_{2}\right)$ constitué des faisceaux localement libres, D'après Ballico ([1]), $M^{0 s}$ est irréductible. Il suffit donc de montrer que $M^{0 s}$ est dense dans $M\left(2,0, c_{2}\right)$. Soit $\mathcal{M}^{0}$ l'ouvert de $\mathcal{M}^{s s}$ constitué des points $(A, B)$ tels que $F_{(A, B)}$ soit localement libre, $M^{0}$ l'image de $\mathcal{M}^{0}$ dans $M\left(2,0, c_{2}\right)$, qui contient $M^{0 s}$. Alors $\mathcal{M}^{0}$ est dense dans $\mathcal{M}^{s s}$ : la démonstration est la même que celle de la proposition (2.8) de [6]. C'est ici que les monades sont utiles. On utilise aussi le fait que pour tout faisceau semi-stable $E$ sur $\mathbb{P}_{1} \times \mathbb{P}_{1}$, et tout point $x$ de $\mathbb{P}_{1} \times \mathbb{P}_{1}$, on a $\operatorname{Ext}^{2}\left(E, E \otimes I_{x}\right)=0$, ce qui est immédiat par dualité de Serre et semi-stabilité de $E$.

Donc $M^{0}$ est dense dans $M\left(2,0, c_{2}\right)$. Il reste donc à prouver que $M^{0 s}$ est dense dans $M^{0}$.

Soit $E$ un fibré vectoriel sur $\mathbb{P}_{1} \times \mathbb{P}_{1}$, dont la classe de $S$-équivalence est un point de $M^{0} \backslash M^{0 s}$. Supposons qu'il soit dans une composante $Z_{k}$ de $M\left(2,0, c_{2}\right) \backslash M^{s}\left(2,0, c_{2}\right)$. Alors il existe des sous-schémas finis $Y, Y^{\prime}$ de $\mathbb{P}_{1} \times \mathbb{P}_{1}$, et une suite exacte

$$
0 \longrightarrow I_{Y}\left(k^{\prime} \alpha,-k^{\prime} \beta\right) \longrightarrow E \longrightarrow I_{Y^{\prime}}\left(-k^{\prime} \alpha, k^{\prime} \beta\right) \longrightarrow 0
$$

avec $k^{\prime}=k$ ou $-k$. On a donc

$$
I_{Y}\left(k^{\prime} \alpha,-k^{\prime} \beta\right) \subset \mathcal{O}\left(k^{\prime} \alpha,-k^{\prime} \beta\right)=I_{Y}\left(k^{\prime} \alpha,-k^{\prime} \beta\right)^{* *} \subset E^{* *}=E,
$$

donc

$$
\mathcal{O}_{Y} \simeq \mathcal{O}\left(k^{\prime} \alpha,-k^{\prime} \beta\right) / I_{Y}\left(k^{\prime} \alpha,-k^{\prime} \beta\right) \subset E / I_{Y}\left(k^{\prime} \alpha,-k^{\prime} \beta\right) \simeq I_{Y^{\prime}}\left(-k^{\prime} \alpha, k^{\prime} \beta\right)
$$

Ceci entraine que $Y=\emptyset$. Par conséquent $k \neq 0$, sinon $l(Y)=\frac{c_{2}}{2}$, et donc $I_{Y}\left(k^{\prime} \alpha,-k^{\prime} \beta\right)$ n'est pas isomorphe à $I_{Y^{\prime}}\left(-k^{\prime} \alpha, k^{\prime} \beta\right)$.

Posons

$$
e_{1}=\operatorname{dim}\left(\operatorname{Ext}^{1}\left(I_{Y}\left(k^{\prime} \alpha,-k^{\prime} \beta\right), I_{Y^{\prime}}\left(-k^{\prime} \alpha, k^{\prime} \beta\right)\right)-1\right.
$$




$$
e_{2}=\operatorname{dim}\left(\operatorname{Ext}^{1}\left(I_{Y^{\prime}}\left(-k^{\prime} \alpha, k^{\prime} \beta\right), I_{Y}\left(k^{\prime} \alpha,-k^{\prime} \beta\right)\right)-1 .\right.
$$

Alors un calcul aisé montre qu'on a, puisque $I_{Y}\left(k^{\prime} \alpha,-k^{\prime} \beta\right)$ n'est pas isomorphe à $I_{Y^{\prime}}\left(-k^{\prime} \alpha, k^{\prime} \beta\right)$, $e_{1}+e_{2}+\operatorname{dim}\left(Z_{k}\right)=4 c_{2}-4$.

Soit $\pi_{0}: \mathcal{M}^{s s} \rightarrow M\left(2,0, c_{2}\right)$ le morphisme quotient. Alors pour tout $(A, B) \in \pi_{0}^{-1}\left(Z_{k} \cap\left(M^{0} \backslash M^{0 s}\right)\right), F_{(A, B)}$ s'écrit comme extension de $I_{Y^{\prime}}\left(-k^{\prime} \alpha, k^{\prime} \beta\right)$ par $I_{Y}\left(k^{\prime} \alpha,-k^{\prime} \beta\right)$. Il en découle aisément que

$$
\begin{aligned}
\operatorname{dim}\left(\pi_{0}^{-1}\left(Z_{k} \cap\left(M^{0} \backslash M^{0 s}\right)\right)\right) & \leq \sup \left(e_{1}, e_{2}\right)+\operatorname{dim}\left(Z_{k}\right)+\operatorname{dim}(G) \\
& \leq e_{1}+e_{2}+\operatorname{dim}\left(Z_{k}\right)+\operatorname{dim}(G) \\
& <4 c_{2}-3+\operatorname{dim}(G)=\operatorname{dim}\left(\mathcal{M}^{s s}\right) .
\end{aligned}
$$

Par conséquent $\operatorname{dim}\left(\pi_{0}^{-1}\left(M^{0} \backslash M^{0 s}\right)\right)<\operatorname{dim}\left(\mathcal{M}^{s s}\right)$, ce qui prouve que $M^{0 s}$ est dense dans $M^{0}$. Ceci achève la démonstration de la proposition 5.3.

5.4 - Points non factoriels de $M\left(2,0, c_{2}\right)$. Les anneaux locaux des points des composantes $Z_{1}, \ldots, Z_{m}$ ne sont pas factoriels, car ces points sont de type 2 . Les anneaux locaux des points de $Z_{0}$ sont factoriels, car les assertions (i) et (ii) de 0.5 sont vraies :

(i) est triviale,

(ii) équivaut à l'irréductibilité des schémas de Hilbert de points de $\mathbb{P}_{1} \times \mathbb{P}_{1}$.

\section{RÉFÉRENCES}

[1] Ballico, E. On moduli of vector bundles on a rational surface. Arch. Math. 49 (1987). 267-272.

[2] Beilinson, A.A. Coherent sheaves on $\mathbb{P}_{n}$ and problems of linear algebra. Funkts. Anal. Prilozh, 12 (1978), 68-69.

[3] Drézet, J.-M. Fibrès exceptionnels et variétés de modules de faisceaux semi-stables sur $\mathbb{P}_{2}(\mathbb{C})$. J. reine angew. Math. 380 (1987), 14-58.

[4] Drézet, J.-M. Groupe de Picard des variétés de modules de faisceaux semi-stables sur $\mathbb{P}_{2}$. Singularities, Representation of Algebras and Vector Bundles. Proc. Lambrecht 1985, Lect. Notes in Math. 1273 (1987), $337-362$.

[5] Drézet, J.-M. Groupe de Picard des variétés de modules de faisceaux semi-stables sur $\mathbb{P}_{2}(\mathbb{C})$. Ann. Inst. Fourier 38 (1988), 105-168.

[6] Drézet, J.-M., Le Potier, J. Fibrés stables et fibrés exceptionnels sur $\mathbb{P}_{2}$. Ann. scient. Éc. Norm. Sup. 18 (1985), 193-244.

[7] Drézet, J.-M., Narasimhan, M.S. Groupe de Picard des variétés de modules de fibrés semi-stables sur une courbe algébrique. Invent. Math. 97 (1989), 53-94.

[8] Fulton, W. Intersection theory. Second edition. Ergeb. der Math. und ihrer Grenzg. Springer-Verlag (1998).

[9] Gieseker, D. On the moduli of vector bundles on an algebraic surface. Ann. of Math. 106 (1977), 45-60.

[10] Grothendieck, A. Technique de descente et théorèmes d'existence en géométrie algébrique, IV Les schémas de Hilbert. séminaire Bourbaki 221, Paris 1960/61.

[11] Horrocks, G. Vector bundles on the punctured spectrum of a local ring. Proc. London Math. Soc. 14 (1964), $689-713$. 
[12] Le Potier, J. Fibrés stables de rang 2 sur $\mathbb{P}_{2}(\mathbb{C})$. Math. Ann. 241 (1979), 217-256.

[13] Maruyama, M. Moduli of stable sheaves II. J. Math. Kyoto Univ. 18 (1978), 557-614.

[14] Maruyama,M. On a compactification of a moduli space or vector bundles on a rational surface. Algebraic Geometry and Commutative Algebra in Honor of M. Nagata, (1987). 233-260.

[15] Matsumura, H. Commutative algebra. New York, W.A. Benjamin Co. (1970).

[16] Mumford, D., Fogarty, J. Geometric invariant theory. Erg. der Math. und ihre Grenzg. Berlin-HeidelbergNew York. Springer (1984).

[17] Narasimhan, M.S., Ramanan, S. Moduli of vector bundles on a compact Riemann surface. Ann. of Math. 89 (1969), 14-51.

[18] Newstead, P.E. Introduction to moduli problems and orbit spaces. TIFR Lect. Notes 51 (I978). 
Note : Ce texte reproduit l'article

Drézet, J.-M. Points non factoriels des variétés de modules de faisceaux semi-stables sur une surface rationnelle. Journ. für die reine und angew. Math. 290 (1991), 99-127.

Soit $X$ une surface rationnelle différente de $\mathbb{P}_{2}$. Il existe alors un morphisme $\phi: X \rightarrow \mathbb{P}_{1}$ dont la fibre générique est isomorphe à $\mathbb{P}_{1}$. Soit $f$ une telle fibre, et supposons que $\left(K_{X}+f\right) \cdot \mathcal{O}_{X}(1)<0$. K. Yoshioka a démontré dans

A note on a paper of J.-M. Drezet on the local factoriality of some moduli spaces. Intern. J. of Math. 7, 6 (1996), 843-858.

que les anneaux locaux des points de type 1 de $M\left(r, c_{1}, c_{2}\right)$ sont factoriels (conjecture 1 de 0.5$)$.

L'article

Drézet, J.-M. Points non factoriels des variétés de modules de faisceaux semi-stables sur une surface rationnelle. Revue Roumaine de math. pures et appl. 36 (1991), 635-645.

contient d'autres résultats qui sont donnés ci-dessous. 


\section{6 - Points FaCtoriels D'Un QUOTIEnT}

Soit $Z$ une variété algébrique irréductible lisse sur $\mathbb{C}$ sur laquelle opère algébriquement un groupe algébrique réductif $G$. On suppose qu'il existe un bon quotient $\pi: Z \rightarrow M$ (au sens un Mumford [16]). La variété $M$ est normale mais non lisse en général. On se propose d'étudier la factorialité des anneaux locaux des points fermés de $M$. On se placera dans le cas suivant : il existe un ouvert $G$-invariant $Z_{0}$ de $\mathrm{Z}$ tel que

$$
\operatorname{codim}_{Z}\left(Z \backslash Z_{0}\right) \geq 2, \quad \pi^{-1}\left(\pi\left(Z_{0}\right)\right)=Z_{0},
$$

que la restriction de $\pi, Z_{0} \rightarrow \pi\left(Z_{0}\right)$ soit un quotient géométrique et que $\mathrm{G}$ agisse librement sur $Z_{0}$. L'ouvert $\pi\left(Z_{0}\right)$ est alors lisse.

L'étude de la factorialité des anneaux locaux des points fermés de $M$ se réduit à celle des $G$-fibrés on droites algébriques sur $Z$. On a en effet le

Théorème 6.1 : Soient $x$ un point fermé de $M, z \in Z$ tel que $\pi(z)=x$ et que l'orbite $G z$ soit fermée. Alors $\mathcal{O}_{x}$ est factoriel si et seulement si pour tout $G$-fibré en droites $L$ sur $Z$, l'action du stabilisateur $G_{z}$ de z sur $L_{z}$ est triviale.

Ceci généralise le théorème A. Il est facile de voir que le groupe des classes d'isomorphisme de G-fibrés en droites sur $Z$ s'identifie naturellement à $\operatorname{Pic}\left(\pi\left(Z_{0}\right)\right)$.

Démonstration. L'anneau $\mathcal{O}_{x}$ est factoriel si et seulement si pour toute hypersurface $Y$ du $M$, le faisceau d'idéaux $I_{Y}$ de $Y$ est libre en $x$. On en déduit l'équivalence des conditions suivantes :

(i) $\mathcal{O}_{x}$ est factoriel.

(ii) Pour tout fibré en droites $L_{0}$ sur $\pi\left(Z_{0}\right)$, il existe un ouvert $U$ de $M$ contenant $\pi\left(Z_{0}\right)$ et $x$, tel que $L_{0}$ se prolonge en un fibré en droites sur $U$.

Montrons que (ii) équivaut à

(iii) Pour tout $G$-fibré en droites $L$ sur $Z, G_{y}$ agit trivialement sur $L_{y}$.

Supposons (ii) vérifiée et soit $L$ un $G$-fibré en droites sur $Z$. Alors $L_{\mid Z_{0}}$ descend à $\pi\left(Z_{0}\right)$. Soit $\Delta=\left(L_{\mid Z_{0}}\right) / G$. D'après (ii), $\Delta$ se prolonge en un fibré en droites $\bar{\Delta}$ sur $U$, voisinage de $x$ contenant $\pi\left(Z_{0}\right)$. Le $G$-fibré en droites $\pi^{*}(\bar{\Delta})$ sur $\pi^{-1}(U)$ coincide avec L sur $Z_{0}$, donc aussi sur $\pi^{-1}(U)$, puisque $\operatorname{codim}_{Z}\left(Z \backslash Z_{0}\right) \geq 2$. Puisque $x \in U, G_{y}$ agit trivialement sur $L_{y}$. Ceci prouve (iii).

Réciproquement, supposons (ii) vraie. Soit $L_{0}$ un fibré en droites sur $\pi\left(Z_{0}\right)$. Alorx $\pi^{*}\left(L_{0}\right)$ est un $G$-fibré en droites sur $Z_{0}$, qui peut se prolonger en un $G$-fibré en droites sur $Z$ (cf. lemme 5.2 de [7]). Soit $U$ l'ouvert de $M$ constitué des points $x^{\prime}$ tels qu'il existe un point $y^{\prime}$ de $\pi^{-1}\left(x^{\prime}\right)$ tel que $G y^{\prime}$ soit fermée et que $G_{y^{\prime}}$ agisse trivialement sur $L_{y^{\prime}}$. Cet ouvert contient $x$. D'après le lemme de descente, $L_{\mid \pi^{-1}(U)}$ descend à $U$, c'est-à-dire qu'il existe un fibré en droites $L^{\prime}$ sur $U$ et un $G$-isomorphisme

$$
L_{\mid \pi^{-1}(U)} \simeq \pi^{*}\left(L^{\prime}\right)
$$

L'ouvert $U$ est un voisinage de $x$ contenant $\pi\left(Z_{0}\right)$ et $L^{\prime}$ est l'extension voulue de $L_{0}$. Ceci prouve (ii).

Ceci achève la démonstration du théorème 6.1 . 
6.1 - Application. Soient $m, n, q$ des entiers positifs, avec $q \geq 3$. On considère l'action évidente de

$$
G=(\mathrm{GL}(m) \times \mathrm{GL}(n)) / \mathbb{C}^{*}
$$

sur $W=L\left(\mathbb{C}^{m} \otimes \mathbb{C}^{q}, \mathbb{C}^{n}\right)$. On en déduit une action de $\operatorname{SL}(m) \times \operatorname{SL}(n)$ sur l'espace projectif $\mathbb{P}=\mathbb{P}(W)$ des droites de $W$. Cette action se prolonge en une action linéaire de $G$ sur $\mathcal{O}_{\mathbb{P}}(1)$. On obtient ainsi une linéarisation de l'action de $G$ sur $\mathbb{P}$. Soient $\mathbb{P}^{s s}$ (resp. $\mathbb{P}^{s}$ ) l'ouvert des points semi-stables (resp. stables) de $\mathbb{P}, W^{s s}$ (resp. $W^{s}$ ) l'ouvert des points de $W$ au dessus de $\mathbb{P}^{s s}$ (resp. $\mathbb{P}^{s}$ ). D'après [3], prop. 15, une application linéaire $\tau: \mathbb{C}^{m} \otimes \mathbb{C}^{q} \rightarrow \mathbb{C}^{n}$ est un point de $W^{s s}\left(\right.$ resp. $\left.W^{s}\right)$ si est seulement si pour tous sous-espaces vectoriels $H_{0}, H_{1}$ de $\mathbb{C}^{m}$, $\mathbb{C}^{n}$ respectivement, tels que $H_{0} \neq\{0\}, H_{1} \neq \mathbb{C}^{n}$, et $\tau\left(H_{0} \otimes \mathbb{C}^{q}\right) \subset H_{1}$, on a

$$
\frac{\operatorname{dim}\left(H_{1}\right)}{\operatorname{dim}\left(H_{0}\right)} \geq \frac{n}{m} \quad(\text { resp. }>) \text {. }
$$

On dit alors que $\tau$ est semi-stable (resp. stable). Il existe un bon quotient par $G$,

$$
\pi: W^{s s} \longrightarrow N(q, m, n) \text {. }
$$

La variété $N(q, m, n)$ est projective et normale. Les groupe $G$ agit librement sur $W^{s}$, et la restriction de $\pi$

$$
W^{s} \longrightarrow \pi\left(W^{s}\right)=N_{s}(q, m, n)
$$

est un quotient géométrique. On montre dans [3] que si $m$ et $n$ ne sont pas premiers entre eux, et si $N(q, m, n)$ n'est pas de dimension 5 , alors $N(q, m, n)$ n'est pas lisse, et l'ouvert de ses points lisses est exactement $N_{s}(q, m, n)$. On a de plus $\operatorname{codim}_{N(q, m, n)}\left(N(q, m, n) \backslash N_{s}(q, m, n)\right) \geq 2$ (les cas où $N(q, m, n)$ est de dimension 5 sont parfaitement connus, et dans ce cas $N(q, m, n)$ est isomorphe à $\left.\mathbb{P}_{5}\right)$.

Soit $\tau: \mathbb{C}^{m} \otimes \mathbb{C}^{q} \rightarrow \mathbb{C}^{n}$ une application linéaire semi-stable. Alors il existe une filtration de Jordan-Hölder de $\tau$, c'est à dire des filtrations

$$
\begin{aligned}
& \{0\}=H_{0}^{0} \subset H_{0}^{1} \subset \cdots \subset H_{0}^{k}=\mathbb{C}^{m}, \\
& \{0\}=H_{1}^{0} \subset H_{1}^{1} \subset \cdots \subset H_{1}^{k}=\mathbb{C}^{n},
\end{aligned}
$$

telles que

$$
\tau\left(H_{0}^{i} \otimes \mathbb{C}^{q}\right) \subset H_{1}^{i} \quad \text { pour } 1 \leq i \leq k,
$$

que l'application induite par $\tau,\left(H_{0}^{i} / H_{0}^{i-1}\right) \otimes \mathbb{C}^{q} \rightarrow H_{1}^{i} / H_{1}^{i-1}$, soit stable, et que

$$
\frac{\operatorname{dim}\left(H_{1}^{i}\right)}{\operatorname{dim}\left(H_{0}^{i}\right)}=\frac{n}{m} .
$$

L'orbite $G \tau$ est fermée dans $W^{s s}$ si et seulement si $\tau$ est scindée, c'est à dire qu'on peut trouver une filtration de Jordan-Hölder telle qu'il existe des sous-espaces vectoriels $K_{0}^{i}$, $K_{1}^{i}$ de $H_{0}^{i}, H_{1}^{i}$ respectivement, pour $1 \leq i \leq k$, tels que

$$
H_{j}^{i}=K_{j}^{i} \oplus H_{j}^{i-1} \quad \text { pour } j=0,1 \quad \text { et } \quad \tau\left(K_{0}^{i} \otimes \mathbb{C}^{q}\right) \subset K_{1}^{i} .
$$


Autrement dit, relativement à des bases convenables de $\mathbb{C}^{m}, \mathbb{C}^{n}, \tau$ correspond à une matrice $m \times m$ d'éléments de $\left(\mathbb{C}^{q}\right)^{*}$, de la forme

$$
\left(\begin{array}{ccccc}
M_{1} & 0 & & & \\
0 & M_{2} & & & \\
& & \cdot & & \\
& & \cdot & & \\
& & & \cdot & \\
& & & & M_{k}
\end{array}\right)
$$

chaque $M_{i}$ représentant la restriction de $\tau, K_{0}^{i} \otimes \mathbb{C}^{q} \rightarrow K_{1}^{i}$.

Posons $p_{j}^{i}=\operatorname{dim}\left(K_{j}^{i}\right)$, pour $1 \leq i \leq k, j=0,1$. Le stabilisateur $G \tau$ de $\tau$ dans $G$ est l'ensemble $\operatorname{des} \mathbb{C}^{*}\left(A_{0}, A_{1}\right)$, où

$$
A_{j}=\left(\begin{array}{cccc}
\lambda_{1} I_{p_{j}^{1}} & & & \\
0 & \cdot & & 0 \\
& & \cdot & \\
& & & \lambda_{k} I_{p_{j}^{k}}
\end{array}\right) \quad \operatorname{pour} j=0,1
$$

où pour tout entier positif $p, I_{p}$ désigne la matrice identité $p \times p$, les $\lambda_{i}$ étant des scalaires non nuls.

Soit $L$ un $G$-fibré en droites sur $W^{s s}$. Puisque $\operatorname{Pic}\left(W^{s s}\right)=0$ et que le groupe des caractères de $G$ est dénombrable, $L$ est le fibré trivial $W^{s s} \times \mathbb{C}$, muni de l'action de $G$ définie par un caractère $\lambda$ de $G$ :

$$
\begin{aligned}
G \times\left(W^{s s} \times \mathbb{C}\right) & \longrightarrow W^{s s} \times \mathbb{C} \\
(g,(z, t)) & \longmapsto(g z, \lambda(g) t) .
\end{aligned}
$$

Le caractère $\lambda$ est de la forme

$$
\begin{aligned}
&(\mathrm{GL}(m) \times \mathrm{GL}(n)) / \mathbb{C}^{*} \longrightarrow \\
& \mathbb{C}^{*}\left(g_{1}, g_{2}\right) \longmapsto \mathbb{C}^{*} \\
& \longrightarrow \operatorname{det}\left(g_{1}\right)^{a} \operatorname{det}\left(g_{2}\right)^{b},
\end{aligned}
$$

$a$ et $b$ étant des entiers tels que $a m+b n=0$.

Il est clair que pour tout $g \in G_{\tau}$ on a $\lambda(g)=1$. Cela découle du fait que $\frac{p_{0}^{i}}{p_{1}^{i}}=\frac{m}{n}$ pour $1 \leq i \leq k$. On en déduit avec le théorème 6.1 la

Proposition 6.2 : La variété $N(q, m, n)$ est localement factorielle.

J.-M. Drézet - Institut de Mathématiques de Jussieu, Case 247, 4 Place Jussieu, F-75252 Paris, FRANCE

E-mail address: jean-marc.drezet@imj-prg.fr 